\title{
Generalized Poisson and Cauchy kernel functions on classical domains
}

\author{
By Kiyosato Окамото, Michiroh Tsukamoтo and Katsuki YокотA \\ (Received December 9, 1998) \\ (from Nagoya Mathematical Journal)
}

\section{Introduction}

In [3], Hua gave the explicit formulas of the Poisson kernel functions and the Cauchy kernel functions for classical domains. In the editor's foreword of the paper [3], Graev says: "In carrying out his investigations by direct computation the author unfortunately does not make use of the possibilities of the group-theoretic aspect of the problems. Yet this group-theoretic aspect would have made possible a clearer understanding of many of the results, and would sometimes have simplified their proofs." Moreover, he gave an example which indicates how the Poisson kernel can be defined in group-theoretic terms.

The purpose of this paper is to give anothor group-theoretic method to obtain the generalized notion which includes, as special cases, not only Poisson kernel functions but also Cauchy kernel functions.

One can easily understand our idea if he studies carefully the simplest example which we give in Section 2 and Section 4.1. In higher dimensional case, however, the computations are not straightforward so that in Section 2.1 we add a heuristic example to explain what is going on in general.

In [5], we already described the above mentioned simplest example.

In [4], we considered the Martin boundary whose isotropy subgroup (at any point) is minimal parabolic.

In this paper we consider the Shilov boundary (whose isotropy subgroup is maximal parabolic) to obtain the Cauchy kernel functions as well as the Poisson kernel functions.

The explicit action of $S O(n, 2)$ on the domain of type IV is quite complicated (see Section 1.4). It took us long time until we finally succeeded in carrying out the computations for the domain of type IV.

Our idea can be roughly explained as follows.

Let $\boldsymbol{D}$ be one of four types of classical domains. For any homogeneous holomorphic line bundle $E$ on $D$ we choose the most general line bundle $L$ (which is 
compatible with $E$ ) on the Shilov boundary. And we define the "canonical" integral transformation from the space of all $C^{\infty}$-sections of $L$ into the space of all $C^{\infty}$ sections of $E$. Choosing $L$ and $E$ suitablly we obtain the Poisson kernel functions and another suitable choice gives us the Cauchy kernel functions.

Finally, we remark that generalized Poisson and Cauchy kernel functions are eigenfunctions of the Laplacian (which is an invariant differential operator) on the classical domain. If we consider vector bundles instead of line bundles we get more general notion of the Poisson kernel functions and the Cauchy kernel functions. It is plausible that those functions may also be eigenfunctions of a certain invariant differential operator on the classical domain. We will show that this is really true in a certain case in the forthcoming paper [6].

\section{Definitions and notation}

Let $G$ be a connected non-compact semi-simple Lie group admitting a finite dimensional faithful representation. Let $K$ be a maximal compact subgroup of $G$. We suppose that $G / K$ is hermitian symmetric. We denote by $\mathfrak{g}$ and $\mathfrak{k}$ the Lie algebras of $G$ and $K$, respectively. Let $\mathfrak{g}_{c}$ be the complexification of $\mathfrak{g}$. We put

$$
\mathfrak{p}=\{Y \in \mathfrak{g} ; B(X, Y)=0 \text { for all } X \in \mathfrak{k}\}
$$

where $B$ denotes the Killing form of the Lie algebra $\mathfrak{g}_{c}$. Then we have

$$
\mathfrak{g}=\mathfrak{k}+\mathfrak{p}, \quad \mathfrak{k} \cap \mathfrak{p}=0, \quad[\mathfrak{p}, \mathfrak{p}] \subset \mathfrak{k}, \quad[\mathfrak{k}, \mathfrak{p}] \subset \mathfrak{p} .
$$

Let $\theta$ be the Cartan involution so that we have

$$
\theta(X+Y)=X-Y \quad(X \in \mathfrak{k}, Y \in \mathfrak{p}) .
$$

For any subset $\mathfrak{m}$ of $\mathfrak{g}_{c}$ we denote by $\mathfrak{m}_{c}$ the complex subspace of $\mathfrak{g}_{c}$ spanned by $\mathfrak{m}$. Since $G / K$ is hermitian symmetric, there exist abelian subalgebras $\mathfrak{p}_{+}$and $\mathfrak{p}_{-}$of $\mathfrak{g}_{c}$ such that

$$
\mathfrak{p}_{c}=\mathfrak{p}_{+}+\mathfrak{p}_{-}, \quad \mathfrak{p}_{+} \cap \mathfrak{p}_{-}=\{0\}, \quad \mathfrak{p}_{+}=\overline{\mathfrak{p}}_{-}, \quad\left[\mathfrak{k}, \mathfrak{p}_{+}\right] \subset \mathfrak{p}_{+}, \quad\left[\mathfrak{k}, \mathfrak{p}_{-}\right] \subset \mathfrak{p}_{-} .
$$

Let $G_{c}$ be the complexification of $G$ with the Lie algebra $\mathfrak{g}_{c}$. We denote by $K_{c}$ (resp. $P_{+}, P_{-}$) the complex analytic subgroup of $G_{c}$ corresponding to $\mathfrak{k}_{c}$ (resp. $\left.\mathfrak{p}_{+}, \mathfrak{p}_{-}\right)$. Then $P_{+} K_{c} P_{-}$is an open subset of $G_{c}$, and any element $w \in P_{+} K_{c} P_{-}$ is uniquely expressed as $w=p_{+} k_{c} p_{-}\left(p_{+} \in P_{+}, k_{c} \in K_{c}, p_{-} \in P_{-}\right)$. This is called the Harish-Chandra decomposition. Put $U=K_{c} P_{-}$. Then $U$ is a complex analytic subgroup of $G_{c}$ and $P_{-}$is a normal subgroup of $U$. Consider the complex homogeneous space $G_{c} / U$. Then $G / K$ can be canonically identified with the open submanifold $G U / U$ of $G_{c} / U$ which is the $G$-orbit of the point $U$ in $G_{c} / U$. 
We introduce an inner product $(\cdot, \cdot)$ on the complex vector space $\mathfrak{p}_{+}$by

$$
\left(z_{1}, z_{2}\right)=B\left(z_{1},-\overline{\theta\left(z_{2}\right)}\right) \quad\left(z_{1}, z_{2} \in \mathfrak{p}_{+}\right)
$$

Then we can prove that $G U \subset P_{+} U$ and that there exists the unique bounded domain $\boldsymbol{D}$ in $\mathfrak{p}_{+}$such that $G U=(\exp \boldsymbol{D}) U$. For any $w$ in $G U$, we denote by $z(w)$ (resp. $u(w))$ the unique element of $\mathfrak{p}_{+}($resp. $U)$ such that $w=(\exp z(w)) u(w)$. For any $g \in G$ and $z \in D$, we denote by $g[z]$ the unique element of $D$ such that $g(\exp z) U=(\exp g[z]) U$. Then we have the canonical onto-isomorphisms:

$$
\begin{array}{ccccc}
G / K & \cong & G U / U & \cong & \boldsymbol{D} \\
U & & \begin{array}{c}
U \\
g K
\end{array} & & \\
& & &
\end{array}
$$

Moreover $G$ acts on $G / K \cong G U / U \cong \boldsymbol{D}$ by the following commutative diagram.

For any $g_{1} \in G$,

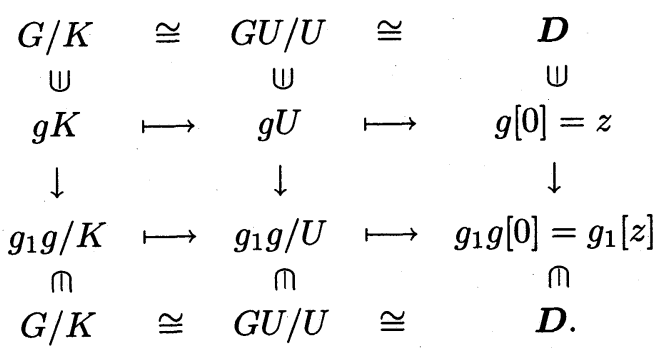

We fix a point $\mu U \in G_{c} / U$ such that $\mu U$ belongs to the boundary of $G U / U$ and that the $G$-orbit of $\mu U$ is compact. Then the $G$-orbit $G \mu U / U$ is the Shilov boundary of the bounded domain $G U / U \cong G / K$ and the isotropy subgroup at the point $\mu U$ of $G_{c} / U$ is a maximal parabolic subgroup of $G$ which we denote by $P$. Put $u_{o}=z(\mu)$ and $\mu_{o}=\exp u_{o}$. Then clearly we get $\mu U=\mu_{o} U=\left(\exp u_{o}\right) U$ which implies that $G \cap \mu U \mu^{-1}=G \cap \mu_{o} U \mu_{o}^{-1}=P$.

Put

$$
\check{\boldsymbol{S}}=\left\{u \in \mathfrak{p}_{+} ; \exp u U \in G \mu_{o} U / U\right\}
$$

Then $\check{\boldsymbol{S}}$ is the Shilov boundary of $\boldsymbol{D}$. For any $g \in G$ and $u \in \check{\boldsymbol{S}}$, we denote by $g[u]$ the unique element of $\check{S}$ such that $g(\exp u) U=(\exp g[u]) U$. Then we have the canonical onto-isomorphisms:

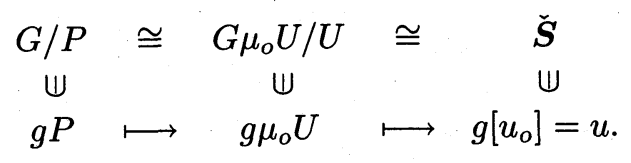


Moreover $G$ acts on $G / P \cong G \mu_{o} U / U \cong \check{S}$ by the following commutative diagram. For any $g_{1} \in G$,

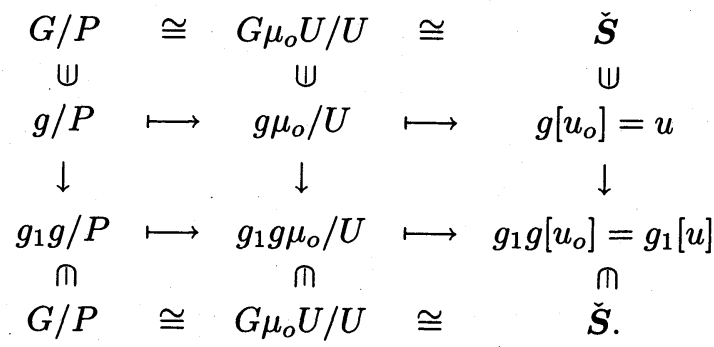
[7]).

Now we need some results on semi-simple Lie groups. (For details, see [1], [2],

Let $P=M A N$ be the Langlands decomposition of $P$ and let $\mathfrak{m}$ (resp. $\mathfrak{a}, \mathfrak{n}$ ) be the Lie algebras corresponding to $M$ (resp. $A, N$ ). Then $M A$ is the centralizer of $A$ in $G$ and $N$ is a normal subgroup of $P$. Moreover $\mathfrak{m}$ is the orthogonal complement of $\mathfrak{a}$ in the centralizer of $\mathfrak{a}$ in $\mathfrak{g}$ with respect to the Killing form.

We denote by $a d$ the adjoint representation of $\mathfrak{g}$ on $\mathfrak{g}$ so that for any $X \in \mathfrak{g}$ we have $\operatorname{ad}(X)(Y)=[X, Y](Y \in \mathfrak{g})$. Let $\rho$ be the linear form on $\mathfrak{a}$ defined by $\rho(H)=\operatorname{Tr}\left(\left.a d(H)\right|_{\mathfrak{n}}\right)$ where $\left.a d(H)\right|_{\mathfrak{n}}$ denotes the restriction of $a d(H)$ to $\mathfrak{n}$. Then one can normalize the Haar measures of $G, K, M, A$ and $N$ in such a way that for any $C^{\infty}$-function $f$ on $G$ with compact support, we have

$$
\int_{G} f(g) d g=\int_{K M A N} f(k m a n) e^{2 \rho(\log a)} d k d m d a d n .
$$

Moreover we can normalize the K-invariant measure on $\check{\boldsymbol{S}}$ such that for any $\boldsymbol{C}^{\infty}$ function $f$ on $\check{\boldsymbol{S}}$, we get

$$
\int_{K} f\left(k\left[u_{o}\right]\right) d k=\int_{\check{S}} f(u) d u .
$$

As usual we denote by $\boldsymbol{N}, \boldsymbol{Z}, \boldsymbol{R}, \boldsymbol{C}, \boldsymbol{C}^{*}$ the set of all natural numbers, the set of all integers, the set of all real numbers, the set of all complex numbers, the set of all non zero complex numbers, respectively.

For any $k \in \boldsymbol{N}$ we denote

$\boldsymbol{R}^{k} \quad$ : the set of all column real vectors of dimension $k$,

$C^{k} \quad:$ the set of all column complex vectors of dimension $k$,

$M_{k}(\boldsymbol{R}) \quad$ : the set of all $(k, k)$ real matrices,

$M_{k}(C)$ : the set of all $(k, k)$ complex matrices,

$G L(k, \boldsymbol{R})$ : the set of all $(k, k)$ real non-singular matrices, 
$G L(k, C)$ : the set of all $(k, k)$ complex non-singular matrices,

$S L(k, \boldsymbol{R}):$ the set of all $(k, k)$ real matrices of determinant one,

$S L(k, C)$ : the set of all $(k, k)$ complex matrices of determinant one,

$O(k) \quad$ : the set of all $(k, k)$ real orthogonal matrices,

$S O(k) \quad$ : the set of all $(k, k)$ real orthogonal matrices of determinant one,

$O(k, C)$ : the set of all $(k, k)$ complex orthogonal matrices,

$S O(k, C)$ : the set of all $(k, k)$ complex orthogonal matrices of determinant one,

$S p(k, \boldsymbol{R})$ : the set of all $(k, k)$ real symplectic matrices,

$S p(k, C)$ : the set of all $(k, k)$ complex symplectic matrices,

$U(k) \quad$ : the set of all $(k, k)$ unitary matrices,

$S U(k) \quad$ : the set of all $(k, k)$ unitary matrices of determinant one,

$\mathfrak{s l}(k, \boldsymbol{R}) \quad$ : the set of all $(k, k)$ real matrices of trace zero,

$\mathfrak{s l}(k, C)$ : the set of all $(k, k)$ complex matrices of trace zero,

$\mathfrak{o}(k) \quad:$ the set of all $(k, k)$ skew symmetric real matrices,

$\mathfrak{o}(k, C) \quad$ : the set of all $(k, k)$ skew symmetric complex matrices,

$I_{k} \quad:$ the identity matrix of degree $k$,

$J_{k} \quad=\left(\begin{array}{cc}0 & I_{k} \\ -I_{k} & 0\end{array}\right)$.

Furthermore, for any $p, q \in \boldsymbol{N}$ we denote

$M_{p, q}(\boldsymbol{R}):$ the set of all $(p, q)$ real matrices,

$M_{p, q}(\boldsymbol{C})$ : the set of all $(p, q)$ complex matrices,

$I_{p, q} \quad=\left(\begin{array}{cc}I_{p} & 0 \\ 0 & -I_{q}\end{array}\right)$

For any matrix $a$ we denote by $\bar{a},{ }^{t} a$ and $a^{*}$ the conjugate, the transpose and the conjugate transpose of $a$, respectively.

For any two hermitian matrices $a, b$ we write $a<<b$ if $b-a$ is positive definite.

We denote the hyperbolic sine and the hyperbolic cosine by sh and ch, respectively:

$$
\operatorname{sh} t=\frac{e^{t}-e^{-t}}{2}, \quad \operatorname{ch} t=\frac{e^{t}+e^{-t}}{2} \quad(t \in \boldsymbol{R})
$$

The reader should notice that in this paper elements of $\boldsymbol{R}^{k}$ and $\boldsymbol{C}^{k}$ are not the row vectors but the column vectors.

For the rest of this paper, we assume that $G$ is simple. Then since $G / P$ is isomorphic to the Shilov boundary, $A$ is one dimensional. 


\subsection{Type I}

Fix any $n, m \in N$ such that $n \geq m$. The bounded symmetric domain of Type $\mathrm{I}$ is defined by

$$
\boldsymbol{D}=\left\{z \in M_{n, m}(\boldsymbol{C}) ; z^{*} z<<I_{m}\right\}
$$

The Shilov boundary of $\boldsymbol{D}$ is given by

$$
\check{\boldsymbol{S}}=\left\{u \in M_{n, m}(\boldsymbol{C}) ; u^{*} u=I_{m}\right\} \text {. }
$$

We define

$$
\begin{aligned}
& G=S U(n, m)=\left\{g \in S L(m+n, C) ; g^{*} I_{n, m} g=I_{n, m}\right\}, \\
& K=\left\{\left(\begin{array}{cc}
k_{1} & 0 \\
0 & k_{2}
\end{array}\right) \in S L(m+n, C) ; k_{1} \in U(n), k_{2} \in U(m)\right\}, \\
& M=\left\{\left(\begin{array}{ccc}
m_{1} & 0 & m_{2} \\
0 & m_{3} & 0 \\
m_{2} & 0 & m_{1}
\end{array}\right) \in G ; \quad \begin{array}{l}
m_{1}, m_{2} \in M_{m}(\boldsymbol{C}) \\
m_{3} \in G L(n-m, \boldsymbol{C})
\end{array},\left|\operatorname{det}\left(m_{1}+m_{2}\right)\right|=1\right\}, \\
& A=\left\{\left(\begin{array}{ccc}
\operatorname{ch} t I_{m} & 0 & \operatorname{sh} t I_{m} \\
0 & I_{n-m} & 0 \\
\operatorname{sh} t I_{m} & 0 & \operatorname{ch} t I_{m}
\end{array}\right) \in G ; t \in \boldsymbol{R}\right\} \\
& N=\left\{\left(\begin{array}{ccc}
I_{m}+\xi-\frac{1}{2} \eta \eta^{*} & \eta & -\xi+\frac{1}{2} \eta \eta^{*} \\
-\eta^{*} & I_{n-m} & \eta^{*} \\
\xi-\frac{1}{2} \eta \eta^{*} & \eta & I_{m}-\xi+\frac{1}{2} \eta \eta^{*}
\end{array}\right) ; \begin{array}{l}
\xi^{*}=-\xi, \xi \in M_{m}(\boldsymbol{C}) \\
\eta \in M_{m, n-m}(\boldsymbol{C})
\end{array}\right\},
\end{aligned}
$$$$
P=M A N .
$$

Then we have $G=K M A N$. Further we define

$$
\begin{aligned}
& G_{c}=S L(m+n, \boldsymbol{C}), \\
& K_{c}=\left\{\left(\begin{array}{cc}
\alpha & 0 \\
0 & \delta
\end{array}\right) \in S L(m+n, \boldsymbol{C}) ; \alpha \in G L(n, \boldsymbol{C}), \delta \in G L(m, \boldsymbol{C})\right\}, \\
& P_{+}=\left\{\left(\begin{array}{cc}
I_{n} & z \\
0 & I_{m}
\end{array}\right) ; z \in M_{n, m}(\boldsymbol{C})\right\}, \\
& P_{-}=\left\{\left(\begin{array}{cc}
I_{n} & 0 \\
w & I_{m}
\end{array}\right) ; w \in M_{m, n}(\boldsymbol{C})\right\},
\end{aligned}
$$




$$
\begin{aligned}
U & =K_{c} P_{-} \\
\mu & =\left(\begin{array}{ccc}
\frac{i}{1+i} I_{m} & 0 & \frac{1}{1+i} I_{m} \\
0 & I_{n-m} & 0 \\
\frac{-i}{1+i} I_{m} & 0 & \frac{1}{1+i} I_{m}
\end{array}\right), \quad \mu_{o}=\left(\begin{array}{ccc}
I_{m} & 0 & I_{m} \\
0 & I_{n-m} & 0 \\
0 & 0 & I_{m}
\end{array}\right) .
\end{aligned}
$$

Then we get

$$
G \cap \mu U \mu^{-1}=G \cap \mu_{o} U \mu_{o}^{-1}=P .
$$

The action of $G$ on $\boldsymbol{D}$ is given by

$$
\boldsymbol{D} \ni z \longmapsto g[z]=(a z+b)(c z+d)^{-1} \in \boldsymbol{D}, \quad g=\left(\begin{array}{ll}
a & b \\
c & d
\end{array}\right) \in G .
$$

We have the canonical onto-isomorphisms:

$$
\begin{array}{cccc}
G / K & \cong & G U / U & \cong \\
\Psi & & \boldsymbol{D} & \Psi \\
g K & \longmapsto & & \Psi U
\end{array}
$$

Moreover the action of $G$ on $\check{S}$ is given by

$$
\check{\boldsymbol{S}} \ni u \longmapsto g[u]=(a u+b)(c u+d)^{-1} \in \check{S}, \quad g=\left(\begin{array}{cc}
a & b \\
c & d
\end{array}\right) \in G
$$

Fix $u_{o}=\left(\begin{array}{c}I_{m} \\ 0\end{array}\right) \in \check{S}$. Then we have the canonical onto-isomorphisms:

$$
\begin{array}{ccccc}
G / P & \cong & G \mu_{o} U / U & \cong & \check{\boldsymbol{S}} \\
\Psi & & \Psi & \Psi \\
g P & \longmapsto & g \mu_{o} U & \longmapsto & g\left[u_{o}\right]=\left(a u_{o}+b\right)\left(c u_{o}+d\right)^{-1} .
\end{array}
$$

\subsection{Type II}

Fix any $n \in N$. The bounded symmetric domain of Type II is defined by

$$
\boldsymbol{D}=\left\{z \in M_{n}(\boldsymbol{C}) ; z^{*} z<<I_{n},{ }^{t} z=z\right\} \text {. }
$$

The Silov boundary of $\boldsymbol{D}$ is given by

$$
\check{\boldsymbol{S}}=\left\{u \in M_{n}(\boldsymbol{C}) ; u^{*} u=I_{n},{ }^{t} u=u\right\} .
$$


We define

$$
\begin{aligned}
\gamma & =\left(\begin{array}{cc}
\frac{i}{1+i} I_{n} & \frac{1}{1+i} I_{n} \\
\frac{-i}{1+i} I_{n} & \frac{1}{1+i} I_{n}
\end{array}\right), \\
G & =\gamma S p(n, \boldsymbol{R}) \gamma^{-1} \\
& =\left\{g \in S p(n, C) ; g^{*} I_{n, n} g=I_{n, n}\right\} \\
& =\left\{\left(\begin{array}{cc}
a & b \\
\bar{b} & \bar{a}
\end{array}\right) \in G ; a, b \in M_{n}(\boldsymbol{C})\right\} \\
K & =\left\{\left(\begin{array}{cc}
k_{1} & 0 \\
0 & k_{1}
\end{array}\right) \in G ; k_{1} \in U(n)\right\}, \\
M & =\left\{\left(\begin{array}{cc}
m_{1} & m_{2} \\
m_{2} & m_{1}
\end{array}\right) \in G ; m_{1}, m_{2} \in M_{n}(\boldsymbol{R}),\left|\operatorname{det}\left(m_{1}+m_{2}\right)\right|=1\right\}, \\
A & =\left\{\left(\begin{array}{cc}
\operatorname{ch} t I_{n} & \operatorname{sh} t I_{n} \\
\operatorname{sh} t I_{n} & \operatorname{ch} t I_{n}
\end{array}\right) ; t \in \boldsymbol{R}\right\}, \\
N & =\left\{\left(\begin{array}{cc}
I_{n}+i \xi & -i \xi \\
i \xi & I_{n}-i \xi
\end{array}\right) ;{ }^{t} \xi=\xi, \xi \in M_{n}(\boldsymbol{R})\right\},
\end{aligned}
$$

Then we have $G=K M A N$. Further we define

$$
\begin{aligned}
& G_{c}=S p(n, C), \\
& K_{c}=\left\{\left(\begin{array}{cc}
\alpha & 0 \\
0 & { }^{t} \alpha^{-1}
\end{array}\right) ; \alpha \in G L(n, C)\right\} \\
& P_{+}=\left\{\left(\begin{array}{cc}
I_{n} & z \\
0 & I_{n}
\end{array}\right) ;{ }^{t} z=z, z \in M_{n}(C)\right\}, \\
& P_{-}=\left\{\left(\begin{array}{cc}
I_{n} & 0 \\
w & I_{n}
\end{array}\right) ;{ }^{t} w=w, w \in M_{n}(C)\right\}, \\
& U=K_{c} P_{-}, \\
& \mu=\left(\begin{array}{cc}
\frac{i}{1+i} I_{n} & \frac{1}{1+i} I_{n} \\
\frac{-i}{1+i} I_{n} & \frac{1}{1+i} I_{n}
\end{array}\right), \quad \mu_{o}=\left(\begin{array}{cc}
I_{n} & I_{n} \\
0 & I_{n}
\end{array}\right), \\
& G \cap \mu U \mu^{-1}=G \cap \mu_{o} U_{\mu_{o}^{-1}}=P .
\end{aligned}
$$


Then we get

$$
G \cap \mu U \mu^{-1}=G \cap \mu_{o} U \mu_{o}^{-1}=P .
$$

The action of $G$ on $D$ is given by

$$
D \ni z \longmapsto g[z]=(a z+b)(\bar{b} z+\bar{a})^{-1} \in D, \quad g=\left(\begin{array}{cc}
a & b \\
\bar{b} & \bar{a}
\end{array}\right) \in G
$$

We have the canonical onto-isomorphisms:

$$
\begin{array}{ccccc}
G / K & \cong & G U / U & \cong & \\
\Psi & & \Psi \\
g K & & & \\
U & g U & \longmapsto & g[0]=b \bar{a}^{-1} .
\end{array}
$$

Moreover the action of $G$ on $\check{\boldsymbol{S}}$ is given by

$$
\check{\boldsymbol{S}} \ni u \longmapsto g[u]=(a u+b)(\bar{b} u+\bar{a})^{-1} \in \check{\boldsymbol{S}}, \quad g=\left(\begin{array}{cc}
a & b \\
\bar{b} & \bar{a}
\end{array}\right) \in G .
$$

Fix $u_{o}=I_{n} \in \check{S}$. Then we have the canonical onto-isomorphisms:

$$
\begin{array}{ccccc}
G / P & \cong & G \mu_{o} U / U & \cong & \check{\boldsymbol{S}} \\
\Psi & & \Psi & \Psi \\
g P & \longmapsto & g \mu_{o} U & \longmapsto g & g\left[u_{o}\right]=(a+b)(\bar{a}+\bar{b})^{-1} .
\end{array}
$$

\subsection{Type III} by

Fix any $n(n>1) \in N$. The bounded symmetric domain of Type III is defined

$$
\boldsymbol{D}=\left\{z \in M_{n}(\boldsymbol{C}) ; z^{*} z<<I_{n},{ }^{t} z=-z\right\}
$$

In case $n$ is even, the Silov boundary of $D$ is given by

$$
\check{\boldsymbol{S}}=\left\{u \in M_{n}(\boldsymbol{C}) ; u^{*} u=I_{n},{ }^{t} u=-u\right\} .
$$

In case $n$ is odd, the Silov boundary of $\boldsymbol{D}$ is given by

$$
\check{\boldsymbol{S}}=\left\{u \in M_{n}(\boldsymbol{C}) ; \operatorname{rank}\left(I_{n}-u^{*} u\right)=1,{ }^{t} u=-u\right\} \text {. }
$$


We put

$$
\begin{aligned}
& \gamma=\left(\begin{array}{cc}
\frac{i}{1+i} I_{n} & \frac{1}{1+i} I_{n} \\
\frac{-i}{1+i} I_{n} & \frac{1}{1+i} I_{n}
\end{array}\right) \\
& S O^{*}(2 n)=\left\{g \in S O(2 n, C) ; g^{*} J_{n} g=J_{n}\right\} \\
& G=\gamma S O^{*}(2 n) \gamma^{-1} \\
& =\left\{g=\left(\begin{array}{cc}
a & b \\
-\bar{b} & \bar{a}
\end{array}\right) ; g \in S U(n, n)\right\}, \\
& K=\left\{\left(\begin{array}{cc}
k_{1} & 0 \\
0 & k_{1}
\end{array}\right) \in S L(2 n, C) ; k_{1} \in U(n)\right\} \text {, } \\
& G_{c}=\left\{g \in S L(2 n, C) ;{ }^{t} g\left(\begin{array}{cc}
0 & I_{n} \\
I_{n} & 0
\end{array}\right) g=\left(\begin{array}{cc}
0 & I_{n} \\
I_{n} & 0
\end{array}\right)\right\}, \\
& K_{c}=\left\{\left(\begin{array}{cc}
\alpha & 0 \\
0 & { }^{t} \alpha^{-1}
\end{array}\right) \in S L(2 n, C) ; \alpha \in G L(n, C)\right\}, \\
& P_{+}=\left\{\left(\begin{array}{cc}
I_{n} & z \\
0 & I_{n}
\end{array}\right) ;{ }^{t} z=-z, z \in M_{n}(C)\right\} \\
& P_{-}=\left\{\left(\begin{array}{cc}
I_{n} & 0 \\
w & I_{n}
\end{array}\right) ;{ }^{t} w=-w, w \in M_{n}(C)\right\} \\
& U=K_{c} P_{-} \text {. }
\end{aligned}
$$

The action of $G$ on $\boldsymbol{D}$ is given by

$$
\boldsymbol{D} \ni z \longmapsto g[z]=(a z+b)(-\bar{b} z+\bar{a})^{-1} \in \boldsymbol{D}, \quad g=\left(\begin{array}{cc}
a & b \\
-\bar{b} & \bar{a}
\end{array}\right) \in G .
$$

We have the canonical onto-isomorphisms:

$$
\begin{aligned}
& G / K \cong G U / U \cong D
\end{aligned}
$$

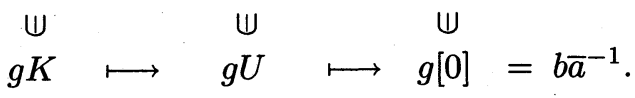

\subsubsection{Case n: even}

Put $m=\frac{n}{2}$. We define

$$
\sigma=\left(\begin{array}{cc}
0 & I_{m} \\
-I_{m} & 0
\end{array}\right)
$$




$$
\begin{aligned}
& M=\left\{g=\left(\begin{array}{cc}
m_{1} & m_{2} \\
-\overline{m_{2}} & \overline{m_{1}}
\end{array}\right) \in G ; \sigma \overline{m_{1}}=m_{1} \sigma, \sigma \overline{m_{2}}=m_{2} \sigma\right. \\
& A=\left\{\left(\begin{array}{cc}
\operatorname{ch} t I_{n} & \operatorname{sh} t \sigma \\
-\operatorname{sh} t \sigma & \operatorname{ch} t I_{n}
\end{array}\right) ; t \in \boldsymbol{R}\right\}, \\
& \left.N=\left\{\left(\begin{array}{cc}
I_{n}+\xi & -\xi \sigma \\
\bar{\xi} \sigma & I_{n}+\bar{\xi}
\end{array}\right) ; \bar{\xi}=\sigma \xi \sigma, m_{1}-m_{2} \sigma\right) \mid=1\right\} \\
& \left.P=M A N, \xi \in M_{n}(\boldsymbol{C})\right\} \\
& \mu=\left(\begin{array}{cc}
\frac{1}{\sqrt{2}} I_{n} & \frac{1}{\sqrt{2}} \sigma \\
\frac{1}{\sqrt{2}} \sigma & \frac{1}{\sqrt{2}} I_{n}
\end{array}\right), \quad \mu_{o}=\left(\begin{array}{cc}
I_{n} & \sigma \\
0 & I_{n}
\end{array}\right)
\end{aligned}
$$

Then we get

$$
G=K M A N, G \cap \mu U \mu^{-1}=G \cap \mu_{o} U \mu_{o}^{-1}=P .
$$

The action of $G$ on $\check{S}$ is given by

$$
\check{\boldsymbol{S}} \ni u \longmapsto g[u]=(a u+b)(-\bar{b} u+\bar{a})^{-1} \in \check{\boldsymbol{S}}, \quad g=\left(\begin{array}{cc}
a & b \\
-\bar{b} & \bar{a}
\end{array}\right) \in G .
$$

Fix $u_{o}=\sigma \in \check{\boldsymbol{S}}$. Then we have the canonical onto-isomorphisms:

$$
\begin{aligned}
& G / P \cong G \mu_{o} U / U \cong \check{\boldsymbol{S}} \\
& \Psi \quad U \quad U \\
& g P \quad \longmapsto g \mu_{o} U \longmapsto g\left[u_{o}\right]=(a \sigma+b)(-\bar{b} \sigma+\bar{a})^{-1} .
\end{aligned}
$$

\subsubsection{Case n: odd}

Fix any $n \in \boldsymbol{N}$. Put $m=\frac{n-1}{2}$. We define

$$
\begin{aligned}
& \omega=\left(\begin{array}{ccc}
I_{m} & 0 & 0 \\
0 & \sqrt{2} & 0 \\
0 & 0 & I_{m}
\end{array}\right), \quad \sigma=\left(\begin{array}{ccc}
0 & 0 & I_{m} \\
0 & 0 & 0 \\
-I_{m} & 0 & 0
\end{array}\right), \\
& M=\left\{g=\left(\begin{array}{cc}
m_{1} & m_{2} \\
-\overline{m_{2}} & \overline{m_{1}}
\end{array}\right) \in G ; \sigma \overline{m_{1}}=m_{1} \sigma, \sigma \overline{m_{2}}=m_{2} \sigma,\right. \\
& A=\left\{\left(\begin{array}{cc}
a(t) & b(t) \\
-b(t) & a(t)
\end{array}\right) ; a(t)=\left(\begin{array}{ccc}
\operatorname{ch} t I_{m} & 0 & 0 \\
0 & 1 & 0 \\
0 & 0 & \operatorname{ch} t I_{m}
\end{array}\right), b(t)=\operatorname{sh} t \sigma, t \in \boldsymbol{R}\right\}
\end{aligned}
$$




$$
\begin{aligned}
N & =\left\{\left(\begin{array}{cc}
I_{n}+\xi & -\xi \sigma \\
\bar{\xi} \sigma & I_{n}+\bar{\xi}
\end{array}\right) ; \bar{\xi}=\sigma \xi \sigma, \xi^{*}=-\xi, \xi \in M_{n}(C)\right\} \\
P & =M A N, \\
\mu & =\left(\begin{array}{cc}
\frac{1}{\sqrt{2}} I_{n} & \frac{1}{\sqrt{2}} \sigma \\
\frac{1}{\sqrt{2}} \sigma & \frac{1}{\sqrt{2}} I_{n}
\end{array}\right), \quad \mu_{o}=\left(\begin{array}{cc}
I_{n} & \sigma \\
0 & I_{n}
\end{array}\right) .
\end{aligned}
$$

Then get

$$
G=K M A N, \quad G \cap \mu U \mu^{-1}=G \cap \mu_{o} U \mu_{o}^{-1}=P .
$$

The action of $G$ on $\check{S}$ is given by

$$
\check{S} \ni u \longmapsto g[u]=(a u+b)(-\bar{b} u+\bar{a})^{-1} \in \check{S}, \quad g=\left(\begin{array}{cc}
a & b \\
-\bar{b} & \bar{a}
\end{array}\right) \in G .
$$

Fix $u_{o}=\sigma \in \check{\boldsymbol{S}}$. Then we have the canonical onto-isomorphisms:

$$
\begin{array}{ccccc}
G / P & \cong & G \mu_{o} U / U & \cong & \check{\boldsymbol{S}} \\
\Psi & & \Psi & \\
g P & \longmapsto g \mu_{o} U & \longmapsto g
\end{array}
$$

\subsection{Type IV}

Fix any $n \in N$. The bounded symmetric domain of Type IV is defined by

$$
\boldsymbol{D}=\left\{z \in \boldsymbol{C}^{n} ; z^{*} z<\frac{1}{2}\left(1+\left|{ }^{t} z z\right|^{2}\right)<1\right\} .
$$

The Shilov boundary of $\boldsymbol{D}$ is given by

$$
\check{\boldsymbol{S}}=\left\{u=e^{i \theta} x \in \boldsymbol{C}^{n} ; 0 \leq \theta<2 \pi, x \in S^{n-1}\right\},
$$

where $S^{n-1}$ denotes the $(n-1)$-dimensional unit sphere:

$$
S^{n-1}=\left\{x \in \boldsymbol{R}^{\mathfrak{n}} ;{ }^{t} x x=1\right\} .
$$

We define

$$
\begin{aligned}
G & =S O(n, 2) \\
& =\left\{g \in S L(n+2, \boldsymbol{R}) ;{ }^{t} g I_{n, 2} g=I_{n, 2}\right\} \\
K & =\left\{\left(\begin{array}{cc}
k_{1} & 0 \\
0 & k_{2}
\end{array}\right) \in S L(n+2, \boldsymbol{R}) ; k_{1} \in O(n), k_{2} \in O(2)\right\} .
\end{aligned}
$$


Let $\widetilde{\Omega}$ denote the set of all $\left(\begin{array}{c}v \\ v_{n+1} \\ v_{n+2}\end{array}\right) \in C^{n+2}\left(v \in C^{n}, v_{n+1}, v_{n+2} \in C\right)$ such that

$$
\begin{aligned}
& { }^{t} v v=v_{n+1}^{2}+v_{n+2}^{2} \\
& v^{*} v<\left|v_{n+1}\right|^{2}+\left|v_{n+2}\right|^{2} \\
& \text { the imaginary part of }\left(\frac{v_{n+1}}{v_{n+2}}\right)>0 .
\end{aligned}
$$

Fix $\omega_{o}=\left(\begin{array}{c}0 \\ i \\ 1\end{array}\right) \in \widetilde{\Omega}$. Let $\Omega$ be the $G$-orbit of the point $\omega_{o}$.

Let $\widetilde{\boldsymbol{D}}$ denote the set of all $\left(\begin{array}{c}w \\ w_{n+1} \\ w_{n+2}\end{array}\right) \in \boldsymbol{C}^{n+2}\left(w \in \boldsymbol{C}^{n}, w_{n+1}, w_{n+2} \in \boldsymbol{C}\right)$ such that

$$
\begin{aligned}
& { }^{t} w w=w_{n+1} w_{n+2} \\
& w^{*} w<\frac{1}{2}\left(\left|w_{n+1}\right|^{2}+\left|w_{n+2}\right|^{2}\right)<\left|w_{n+2}\right|^{2}
\end{aligned}
$$

Put

$$
\gamma=\left(\begin{array}{cc}
I_{n} & 0 \\
0 & \left(\begin{array}{cc}
i & 1 \\
-i & 1
\end{array}\right)
\end{array}\right)
$$

Then $\boldsymbol{G}$ acts on $\boldsymbol{D}$ transitively by the following commutative diagram.

For any $g=\left(\begin{array}{ll}a & b \\ c & d\end{array}\right) \in G$

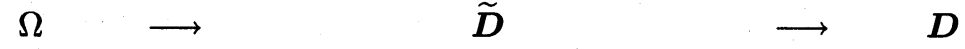

$$
\begin{aligned}
& \text { U } U \text { U } \\
& \left(\begin{array}{c}
v \\
v_{n+1} \\
v_{n+2}
\end{array}\right) \longmapsto \gamma\left(\begin{array}{c}
v \\
v_{n+1} \\
v_{n+2}
\end{array}\right)=\left(\begin{array}{c}
w \\
w_{n+1} \\
w_{n+2}
\end{array}\right) \quad \longmapsto w / w_{n+2}=z \\
& \downarrow \downarrow \downarrow \downarrow \\
& g\left(\begin{array}{c}
v \\
v_{n+1} \\
v_{n+2}
\end{array}\right) \longmapsto \gamma g\left(\begin{array}{c}
v \\
v_{n+1} \\
v_{n+2}
\end{array}\right)=\gamma g \gamma^{-1}\left(\begin{array}{c}
w \\
w_{n+1} \\
w_{n+2}
\end{array}\right) \longmapsto \quad g[z] \\
& \text { m } \mathrm{m} \text { m } \\
& \begin{array}{llllll} 
& \widetilde{D} & \longrightarrow & \longrightarrow & D
\end{array}
\end{aligned}
$$


where

$$
g[z]=\left(a z+b\left(\begin{array}{c}
\frac{i}{2}\left(1-{ }^{t} z z\right) \\
\frac{1}{2}\left(1+{ }^{t} z z\right)
\end{array}\right)\right)\left((-i, 1)\left(c z+d\left(\begin{array}{c}
\frac{i}{2}\left(1-{ }^{t} z z\right) \\
\frac{1}{2}\left(1+{ }^{t} z z\right)
\end{array}\right)\right)\right)^{-1}
$$

In particular we get

$$
\begin{aligned}
& \begin{array}{llll}
\Omega & \begin{array}{c}
\widetilde{D} \\
\Psi
\end{array} & \longrightarrow & \boldsymbol{D} \\
\Psi
\end{array} \\
& \omega_{o} \longmapsto \quad \gamma \omega_{o}=\left(\begin{array}{l}
0 \\
0 \\
2
\end{array}\right) \quad \longmapsto \quad 0 \\
& \downarrow \downarrow \downarrow \\
& g \omega_{o} \longmapsto \gamma g \omega_{o}=\left(\begin{array}{c}
b\left(\begin{array}{c}
i \\
1
\end{array}\right) \\
(i, 1) d\left(\begin{array}{c}
i \\
1
\end{array}\right) \\
(-i, 1) d\left(\begin{array}{c}
i \\
1
\end{array}\right)
\end{array}\right) \longmapsto g[0]=z \\
& \begin{array}{llll}
\mathrm{m} & \mathrm{m} & & \mathrm{m} \\
\Omega & \widetilde{D} & \boldsymbol{D}
\end{array}
\end{aligned}
$$

Thus we get

$$
\begin{aligned}
& \left(b\left(\begin{array}{c}
i \\
1
\end{array}\right)\right)\left((-i, 1) d\left(\begin{array}{c}
i \\
1
\end{array}\right)\right)^{-1}=z \\
& \left((i, 1) d\left(\begin{array}{c}
i \\
1
\end{array}\right)\right)\left((-i, 1) d\left(\begin{array}{c}
i \\
1
\end{array}\right)\right)^{-1}={ }^{t} z z .
\end{aligned}
$$

We denote by $\partial \widetilde{\Omega}$ and $\partial \widetilde{\boldsymbol{D}}$ the boundary of $\widetilde{\Omega}$ and $\widetilde{\boldsymbol{D}}$, respectively. Fix $p_{o}=\left(\begin{array}{l}x_{o} \\ y_{o}\end{array}\right) \in \partial \widetilde{\Omega},\left(x_{o}=\left(\begin{array}{l}1 \\ 0\end{array}\right) \in C^{n}, y_{o}=\left(\begin{array}{l}0 \\ 1\end{array}\right) \in C^{2}\right)$. Let $\partial \Omega_{p_{o}}$ be the $G$-orbit of the point $p_{o}$. Then $G$ acts on $\check{S}$ transitively by the following commutative diagram. 
For any $g \in G$

$$
\begin{aligned}
& \partial \Omega_{p_{o}} \quad \longrightarrow \\
& \partial \widetilde{\boldsymbol{D}} \\
& \longrightarrow \quad \check{S} \\
& \Psi \\
& \Psi \\
& \left(\begin{array}{c}
v \\
v_{n+1} \\
v_{n+2}
\end{array}\right) \longmapsto \gamma\left(\begin{array}{c}
v \\
v_{n+1} \\
v_{n+2}
\end{array}\right)=\left(\begin{array}{c}
w \\
w_{n+1} \\
w_{n+2}
\end{array}\right) \quad \longmapsto w / w_{n+2}=u \\
& \downarrow \downarrow \downarrow \\
& g\left(\begin{array}{c}
v \\
v_{n+1} \\
v_{n+2}
\end{array}\right) \longmapsto \gamma g\left(\begin{array}{c}
v \\
v_{n+1} \\
v_{n+2}
\end{array}\right)=\gamma g \gamma^{-1}\left(\begin{array}{c}
w \\
w_{n+1} \\
w_{n+2}
\end{array}\right) \longmapsto \quad g[u]
\end{aligned}
$$

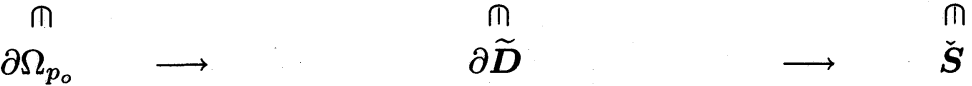

where

$$
g[u]=\left(a u+b\left(\begin{array}{c}
\frac{i}{2}\left(1-{ }^{t} u u\right) \\
\frac{i}{2}\left(1+{ }^{t} u u\right)
\end{array}\right)\right)\left((-i, 1)\left(c u+d\left(\begin{array}{c}
\frac{i}{2}\left(1-{ }^{t} u u\right) \\
\frac{i}{2}\left(1+{ }^{t} u u\right)
\end{array}\right)\right)\right)^{-1} \cdot
$$

Put $x_{o}=\left(\begin{array}{l}1 \\ 0\end{array}\right) \in \boldsymbol{R}^{n}$ and $y_{o}=\left(\begin{array}{l}0 \\ 1\end{array}\right) \in \boldsymbol{R}^{2}$. Let $k=\left(\begin{array}{cc}k_{1} & 0 \\ 0 & k_{2}\end{array}\right)$, $k_{2}=k_{\theta}=\left(\begin{array}{cc}\cos \theta & \sin \theta \\ -\sin \theta & \cos \theta\end{array}\right)$ and denote $k_{1} x_{o}=x \in \boldsymbol{S}^{n-1}$. Then we get

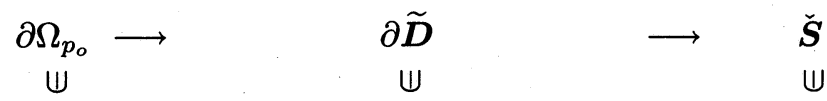

$$
\begin{aligned}
& p_{o} \longmapsto \quad \gamma p_{o}=\left(\begin{array}{c}
x_{o} \\
1 \\
1
\end{array}\right) \quad \longmapsto \quad x_{o} \\
& \downarrow \downarrow \downarrow \downarrow \downarrow \\
& k p_{o} \longmapsto \gamma k \gamma^{-1}\left(\begin{array}{c}
x_{o} \\
1 \\
1
\end{array}\right)=\left(\begin{array}{c}
x \\
e^{i \theta} \\
e^{-i \theta}
\end{array}\right) \longmapsto k\left[x_{o}\right]=u=e^{i \theta} x
\end{aligned}
$$

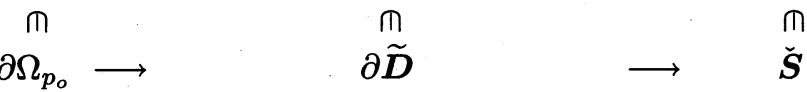

We define

$$
M=\left\{\left(\begin{array}{ccc}
m_{1} & 0 & 0 \\
0 & m_{2} & 0 \\
0 & 0 & m_{1}
\end{array}\right) ; m_{1} \in O(1), m_{2} \in S O(n-1,1)\right\}
$$




$$
\begin{aligned}
& A=\left\{\left(\begin{array}{ccc}
\operatorname{ch} t & 0 & \operatorname{sh} t \\
0 & I_{n} & 0 \\
\operatorname{sh} t & 0 & \operatorname{ch} t
\end{array}\right) \in G ; t \in \boldsymbol{R}\right\} \\
& N=\left\{\left(\begin{array}{ccc}
1+\xi-\frac{1}{2} \eta^{t} \eta & \eta & -\xi+\frac{1}{2} \eta^{t} \eta \\
-{ }^{t} \eta & I_{n} & { }^{t} \eta \\
\xi-\frac{1}{2} \eta^{t} \eta & \eta & 1-\xi+\frac{1}{2} \eta^{t} \eta
\end{array}\right) ; \xi \in \boldsymbol{R},{ }^{t} \eta \in \boldsymbol{R}^{n}\right\} \\
& G=K M A N, \quad P=M A N, \\
& G_{c}=S O(n+2, C) \text {, } \\
& \widetilde{K}_{c}=\left\{\left(\begin{array}{ccc}
\alpha & 0 & 0 \\
0 & \delta_{1} & 0 \\
0 & 0 & \delta_{2}
\end{array}\right) \in S L(n+2, \boldsymbol{C}) ; \alpha \in S O(n, \boldsymbol{C}), \delta_{1}, \delta_{2} \in \boldsymbol{C}^{*}\right\}, \\
& \widetilde{P}_{+}=\left\{\left(\begin{array}{ccc}
I_{n} & 0 & z \\
-2^{t} z & 1 & -{ }^{t} z z \\
0 & 0 & 1
\end{array}\right) ; z \in C^{n}\right\} \\
& \widetilde{P}_{-}=\left\{\left(\begin{array}{ccc}
I_{n} & w & 0 \\
0 & 1 & 0 \\
-2^{t} w & -{ }^{t} w w & 1
\end{array}\right) ; w \in C^{n}\right\} \\
& \widetilde{U}=\widetilde{K}_{c} \widetilde{P}_{-}, \\
& K_{c}=\gamma^{-1} \widetilde{K}_{c} \gamma, P_{+}=\gamma^{-1} \widetilde{P}_{+} \gamma, \quad P_{-}=\gamma^{-1} \widetilde{P}_{-} \gamma, \\
& \mu=\left(\begin{array}{ccc}
\frac{i}{1+i} & 0 & \frac{1}{1+i} \\
0 & I_{n} & 0 \\
\frac{-i}{1+i} & 0 & \frac{1}{1+i}
\end{array}\right) \gamma, \quad \mu_{o}=\left(\begin{array}{ccc}
1 & 0 & 1 \\
0 & I_{n} & 0 \\
0 & 0 & 1
\end{array}\right) \gamma \\
& G \cap \mu U \mu^{-1}=G \cap \mu_{o} U \mu_{o}^{-1}=P, \\
& \check{\boldsymbol{S}}=\left\{u=e^{i \theta} x \in \boldsymbol{C}^{n} ; 0 \leq \theta<2 \pi, x \in S^{n-1}\right\} \text {. }
\end{aligned}
$$

We have the canonical onto-isomorphisms

$$
\begin{aligned}
G / K \cong G U / U & \cong \boldsymbol{D} \\
\Psi & \mathbb{U} \\
g K & \longmapsto g U
\end{aligned}
$$


Fix $u_{o}=\left(\begin{array}{l}1 \\ 0\end{array}\right) \in \check{\boldsymbol{S}}$. Then we have the canonical onto-isomorphisms:

$$
\begin{aligned}
& G / P \cong G \mu_{o} U / U \cong \check{\boldsymbol{S}} \\
& \Psi \quad \Psi \quad U \\
& g P \longmapsto g \mu_{o} U \longmapsto g\left[u_{o}\right]=\left(a u_{o}+b\left(\begin{array}{l}
0 \\
1
\end{array}\right)\right)\left((-i, 1)\left(c u_{o}+d\left(\begin{array}{l}
0 \\
1
\end{array}\right)\right)\right)^{-1} \text {. }
\end{aligned}
$$

\section{Heuristic examples}

We denote by $\boldsymbol{D}$ the unit open disk and by $\partial \boldsymbol{D}$ the boundary of $\boldsymbol{D}$ :

$$
\begin{aligned}
& \boldsymbol{D}=\{z \in \boldsymbol{C} ;|z|<1\} \\
& \partial \boldsymbol{D}=\{z \in \boldsymbol{C} ;|z|=1\} \\
& \overline{\boldsymbol{D}}=\boldsymbol{D} \cup \partial \boldsymbol{D} .
\end{aligned}
$$

Let $F$ be a harmonic function on $\boldsymbol{D}$ which is extended to a continuous function on $\bar{D}$ and let $\Phi$ denote the boundary value of $F$. Then as is well-known $F$ is uniquely determined by $\Phi$ and given by the Poisson integral

$$
F(z)=\frac{1}{2 \pi} \int_{0}^{2 \pi} \frac{1-|z|^{2}}{\left|e^{i \theta}-z\right|^{2}} \Phi\left(e^{i \theta}\right) d \theta \quad(z \in D) .
$$

In the following, for any $z \in \boldsymbol{C}$ we denote $z=x+i y(x, y \in \boldsymbol{R})$. Differentiating a complex power of the Poisson kernel function by the Laplacian we get

$$
\left(\frac{\partial^{2}}{\partial x^{2}}+\frac{\partial^{2}}{\partial y^{2}}\right)\left(\frac{1-|z|^{2}}{\left|e^{i \theta}-z\right|^{2}}\right)^{s}=\frac{4 s(s-1)}{\left(1-|z|^{2}\right)^{2}}\left(\frac{1-|z|^{2}}{\left|e^{i \theta}-z\right|^{2}}\right)^{s} \quad(s \in C) .
$$

This shows that the complex power of the Poisson kernel function is not an eigenfunction of the Laplacian. Multiplying $\left(1-|z|^{2}\right)^{2}$ to both sides of the above equation we have

$$
\left(1-|z|^{2}\right)^{2}\left(\frac{\partial^{2}}{\partial x^{2}}+\frac{\partial^{2}}{\partial y^{2}}\right)\left(\frac{1-|z|^{2}}{\left|e^{i \theta}-z\right|^{2}}\right)^{s}=4 s(s-1)\left(\frac{1-|z|^{2}}{\left|e^{i \theta}-z\right|^{2}}\right)^{s}
$$

Thus the complex power of the Poisson kernel function is not an eigenfunction of $\left(\frac{\partial^{2}}{\partial x^{2}}+\frac{\partial^{2}}{\partial y^{2}}\right)$ but an eigenfunction of $\left(1-|z|^{2}\right)^{2}\left(\frac{\partial^{2}}{\partial x^{2}}+\frac{\partial^{2}}{\partial y^{2}}\right)$ which happens to be the Laplacian of a non Euclidean geometry.

Put

$$
G=S U(1,1)=\left\{\left(\begin{array}{cc}
a & b \\
\bar{b} & \bar{a}
\end{array}\right) ;|a|^{2}-|b|^{2}=1\right\}
$$


Then $G$ acts transitively on $\boldsymbol{D}$ as follows.

For any $g=\left(\begin{array}{cc}a & b \\ \bar{b} & \bar{a}\end{array}\right) \in G$

$$
\boldsymbol{D} \ni z \longmapsto g[z] \in \boldsymbol{D}, \quad g[z]=\frac{a z+b}{\bar{b} z+\bar{a}}
$$

The above differential operator $\left(1-|z|^{2}\right)^{2}\left(\frac{\partial^{2}}{\partial x^{2}}+\frac{\partial^{2}}{\partial y^{2}}\right)$ is nothing but the Laplacian defined by the $G$-invariant riemannian metric $\frac{1}{\left(1-|z|^{2}\right)^{2}}\left(d x^{2}+d y^{2}\right)$ which is the riemannian metric of the non Euclidean geometry of hyperbolic type.

In case $s=1$ the above eigenvalue $4 s(s-1)$ is equal to 0 so that the Poisson kernel function is also an eigenfunction of $\left(1-|z|^{2}\right)^{2}\left(\frac{\partial^{2}}{\partial x^{2}}+\frac{\partial^{2}}{\partial y^{2}}\right)$.

In this way we have found the notion of the generalized Poisson integral whose kernel function is a complex power of the Poisson kernel function. It is plausible that this notion would be something to do with the non Euclidean geometry corresponding to the action of the group $G=S U(1,1)$ on $D$.

Now the isotropy subgroup of $G$ at the point $0 \in \boldsymbol{D}$ is given by

$$
K=\left\{k_{\theta} ; 0 \leq \theta<4 \pi\right\}, \quad k_{\theta}=\left(\begin{array}{cc}
e^{i \theta / 2} & 0 \\
0 & e^{-i \theta / 2}
\end{array}\right)
$$

so that we get an onto-isomorphism:

$$
G / K \ni g K=\left(\begin{array}{cc}
a & b \\
\bar{b} & \bar{a}
\end{array}\right) K \longmapsto \frac{b}{\bar{a}}=z \in \boldsymbol{D} .
$$

On the other hand the group $G$ acts transitively on $\partial \boldsymbol{D}$ as follows.

For any $g=\left(\begin{array}{cc}a & b \\ \bar{b} & \bar{a}\end{array}\right) \in G$

$$
\partial \boldsymbol{D} \ni e^{i \theta} \longmapsto g\left[e^{i \theta}\right] \in \partial \boldsymbol{D}, \quad g\left[e^{i \theta}\right]=\frac{a e^{i \theta}+b}{\bar{b} e^{i \theta}+\bar{a}} .
$$

We denote by $P$ the isotropy subgroup of $G$ at the point $1 \in \partial D$. Then we get an onto-isomorphism:

$$
G / P \ni g P=\left(\begin{array}{cc}
a & b \\
\bar{b} & \bar{a}
\end{array}\right) P \longmapsto g[1]=\frac{a+b}{\bar{a}+\bar{b}}=e^{i \theta} \in \boldsymbol{D} .
$$

Notice that the Poisson integral defines a mapping from $C^{\infty}(G / P) \cong C^{\infty}(\partial D)$ to $\boldsymbol{C}^{\infty}(\boldsymbol{D}) \cong \boldsymbol{C}^{\infty}(G / K)$. Let $\phi$ be any element of $\boldsymbol{C}^{\infty}(G / P)$. Then $\phi$ can be regarded as a $C^{\infty}$-function on $G$ which is invariant by the right action of $P$. The 
most canonical way to assign a function $f \in C^{\infty}(G / K)$ (which is regarded as a function on $G$ invariant by the right action of $K$ ) to $\phi$ is given by the Weyl's unitary trick:

$$
f(g)=\int_{K} \phi(g k) d k=\frac{1}{4 \pi} \int_{0}^{4 \pi} \phi\left(g k_{\theta}\right) d \theta
$$

where $d k$ denotes the normalized Haar measure of $K$. In the following we prove that this simple mapping:

$$
\mathcal{P}: \boldsymbol{C}^{\infty}(G / P) \ni \phi \longmapsto f \in \boldsymbol{C}^{\infty}(G / K)
$$

gives us the Poisson integral.

Let $f=\mathcal{P}(\phi)$. Then for any $g \in G$ we have

$$
f(g)=\frac{1}{4 \pi} \int_{0}^{4 \pi} \phi\left(g k_{\varphi}\right) d \varphi
$$

For $g=\left(\begin{array}{cc}a & b \\ \bar{b} & \bar{a}\end{array}\right)$, we put

$$
g k_{\varphi}=k_{\theta} p \quad\left(k_{\theta} \in K, p \in P\right)
$$

Then we have

$$
g k_{\varphi}[1]=k_{\theta} p[1]
$$

which implies that

$$
\frac{a e^{i \varphi}+b}{\bar{b} e^{i \varphi}+\bar{a}}=e^{i \theta} .
$$

Then we get

$$
e^{i \varphi}=\frac{\bar{a} e^{i \theta}-b}{-\bar{b} e^{i \theta}+a}
$$

Thus

$$
\frac{d \varphi}{d \theta}=\frac{1}{\left|\bar{a} e^{i \theta}-b\right|^{2}}=\frac{1-|z|^{2}}{\left|e^{i \theta}-z\right|^{2}}
$$

On the other hand, we see that

$$
f(g)=f\left(\frac{b}{\bar{a}}\right)=F(z) .
$$


Therefore we finally obtain: for any $z \in \boldsymbol{D}$

$$
\begin{aligned}
F(z) & =f(g) \\
& =\frac{1}{4 \pi} \int_{0}^{4 \pi} \phi\left(g k_{\varphi}\right) d \varphi \\
& =\frac{1}{4 \pi} \int_{0}^{4 \pi} \phi\left(k_{\theta}\right)\left|\frac{d \varphi}{d \theta}\right| d \theta \\
& =\frac{1}{4 \pi} \int_{0}^{4 \pi} \frac{1-|z|^{2}}{\left|e^{i \theta}-z\right|^{2}} \Phi\left(e^{i \theta}\right) d \theta \\
& =\frac{1}{2 \pi} \int_{0}^{2 \pi} \frac{1-|z|^{2}}{\left|e^{i \theta}-z\right|^{2}} \Phi\left(e^{i \theta}\right) d \theta
\end{aligned}
$$

This is nothing but the usual Poisson integral.

To obtain the above mentioned generalized Poisson integral we consider line bundles over $G / P$ which we explain in Section 4.1 .

\section{1. $S U(n, 1)$}

Let us take and fix an element $g=\left(\begin{array}{ll}a & b \\ c & d\end{array}\right) \in S U(n, 1)$ which gives rise to an action to be considered.

For each vector $u={ }^{t}\left(u_{1}, u_{2}, \ldots, u_{n}\right) \in C^{n}$ with $u^{*} u=1$, we define the action of $g$ :

$$
v={ }^{t}\left(v_{1}, v_{2}, \ldots, v_{n}\right)=g[u]=(a u+b)(c u+d)^{-1} .
$$

Here, we should like to verify by direct calculation that the Jacobian of this mapping $u \longrightarrow v$ is

$$
\frac{1}{|c u+d|^{2 n}}
$$

In what follows, we see how it came about.

Incidentally, the absence of ' $a$ ' and ' $b$ ' may look somewhat curious. But recall that we do have the relation $g^{*} I_{n, 1} g=I_{n, 1}$ as well as $\operatorname{det} g=1$ which tell us ' $a$ ', ' $b$ ', ' $c$ ' and ' $d$ ' are closely related to each other and there is nothing strange. To say one more word, it follows from the above relations

$$
g^{-1}=I_{n, 1} g^{*} I_{n, 1}
$$

Hence

$$
\frac{1}{\operatorname{det} g}\left(\widehat{g}_{j i}\right)=\left(\widehat{g}_{j i}\right)=\left(\begin{array}{cc}
\left(\widehat{a}_{j i}\right) & \left(\widehat{c_{j}}\right) \\
\left(\widehat{b_{i}}\right) & \widehat{d}
\end{array}\right)=\left(\begin{array}{cc}
a^{*} & -c^{*} \\
-b^{*} & \bar{d}
\end{array}\right)
$$


where $\widehat{g}_{j i}$ denotes the $(i, j)$-cofacor of a matrix $\left(g_{i j}\right)$. In other words, we have sufficient informations about the inverse matrix of $g$ or, cofactors of $g$.

Let $A=\left(a_{i j}\right), B=\left(b_{i j}\right) \in M_{n}(C)$. If $A$ has the same property as $g$ noticed above:

$$
{ }^{t} A^{-1}=B, \quad \operatorname{det} A=\operatorname{det} B=1,
$$

it follows that

$$
\begin{aligned}
\widehat{b}_{i j} & =(j, i) \text {-component of } B^{-1} \\
& =(j, i) \text {-component of }{ }^{t} \\
& =a_{i j}
\end{aligned}
$$

This fact turns out to be fairly important, later.

Before executing calculations, we prepare a few notations:

$$
\begin{aligned}
& t={ }^{t}\left(t_{1}, t_{2}, \ldots, t_{n}\right) \in \boldsymbol{R}^{n}, \quad t_{j}=s_{j} \cos \theta_{j}, \\
& s_{j}=\sin \theta_{o} \sin \theta_{1} \cdots \sin \theta_{j-1},(j=1, \ldots, n) \text { and } \theta_{o}-\pi / 2=\theta_{n}=0 .
\end{aligned}
$$

Moving around on the sphere $S^{n-1}$, the vector $t$ is perpendicular to each $\frac{\partial t}{\partial \theta_{j}}$ $(j=1, \ldots, n-1)$.

Put $w=t, \frac{\partial t}{\partial \theta_{j}}=s_{j} w_{j}(j=1, \ldots, n-1)$. Then we have an orthonormal system $\left\{w, w_{1}, w_{2}, \ldots, w_{n-1}\right\}$.

We take spherical coordinates

$(r, \psi)=(r, \varphi, \theta)=\left(r, \varphi_{1}, \varphi_{2}, \ldots, \varphi_{n}, \theta_{1}, \theta_{2}, \ldots, \theta_{n-1}\right)$ for $\boldsymbol{C}^{n}$ as follows:

$$
z_{j}=r e^{i \varphi_{j}} t_{j}, \quad(j=1, \ldots, n)
$$

with $t_{j}, s_{j}, \theta_{o}$ and $\theta_{n}$ as above. Here $z=\left(z_{1}, z_{2}, \ldots, z_{n}\right)$ is the system of coordinates for $\boldsymbol{C}^{n}$, as usual.

Let $z_{j}$ be equal to $x_{j}+i x_{n+j},\left(x_{j}, x_{n+j} \in \boldsymbol{R}\right)$. Then it holds $x_{j}=r \cos \varphi_{j} t_{j}$, $x_{n+j}=r \sin \varphi_{j} t_{j}$, so that

$$
\begin{aligned}
\mathrm{d} x_{1} \wedge \mathrm{d} x_{2} \wedge \cdots \wedge \mathrm{d} x_{2 n} \\
\quad=r^{2 n-1} J(\psi) \mathrm{d} r \wedge \mathrm{d} \varphi_{1} \wedge \cdots \wedge \mathrm{d} \varphi_{n} \wedge \mathrm{d} \theta_{1} \wedge \cdots \wedge \mathrm{d} \theta_{n-1}
\end{aligned}
$$

where we put

$$
\begin{aligned}
J(\psi) & =s_{1} s_{2} \cdots s_{n-1} t_{1} t_{2} \cdots t_{n} \\
& =\sin ^{2 n-3} \theta_{1} \sin ^{2 n-5} \theta_{2} \cdots \sin \theta_{n-1} \cos \theta_{1} \cos \theta_{2} \cdots \cos \theta_{n-1}
\end{aligned}
$$


Taking $r$ to be equal to 1 , we have the invariant measure on $S^{2 n-1}$ :

$$
J(\psi) \mathrm{d} \varphi_{1} \wedge \cdots \wedge \mathrm{d} \varphi_{n} \wedge \mathrm{d} \theta_{1} \wedge \cdots \wedge \mathrm{d} \theta_{n-1}
$$

In order to calculate the Jacobian of (1), we use some more notations:

$$
\begin{aligned}
u_{j} & =e^{i \varphi_{j}} t_{j}, \quad t_{j}=t(\theta)_{j}=s(\theta)_{j} \cos \theta_{j} \\
s_{j} & =s(\theta)_{j}=\sin \theta_{o} \sin \theta_{1} \cdots \sin \theta_{j-1}, \quad \theta_{o}-\pi / 2=\theta_{n}=0 \\
v_{j} & =e^{i \varphi_{j}^{\prime}} t_{j}^{\prime}, \quad t_{j}^{\prime}=t\left(\theta^{\prime}\right)_{j}=s\left(\theta^{\prime}\right)_{j} \cos \theta_{j}^{\prime} \\
s_{j}^{\prime} & =s\left(\theta^{\prime}\right)_{j}=\sin \theta_{o}^{\prime} \sin \theta_{1}^{\prime} \cdots \sin \theta_{j-1}^{\prime}, \quad \theta_{o}^{\prime}-\pi / 2=\theta_{n}^{\prime}=0 \\
\psi & =(\varphi, \theta)=\left(\varphi_{1}, \varphi_{2}, \ldots, \varphi_{n}, \theta_{1}, \theta_{2}, \ldots, \theta_{n-1}\right) \\
\psi^{\prime} & =\left(\varphi^{\prime}, \theta^{\prime}\right)=\left(\varphi_{1}^{\prime}, \varphi_{2}^{\prime}, \ldots, \varphi_{n}^{\prime}, \theta_{1}^{\prime}, \theta_{2}^{\prime}, \ldots, \theta_{n-1}^{\prime}\right)
\end{aligned}
$$

Conforming to what our predecessors taught us, we make use of

$$
\widetilde{v}={ }^{t}\left(v_{1}, \ldots, v_{n}, \overline{v_{1}}, \ldots, \overline{v_{n-1}}\right) \text {. }
$$

Now, we are to make sure of the relation

$$
\frac{J(\psi)}{J\left(\psi^{\prime}\right)} \operatorname{det}\left(\frac{\partial \psi^{\prime}}{\partial \psi}\right)=\frac{1}{|c u+d|^{2 n}}
$$

where, $J(\psi)=\sin ^{2 n-3} \theta_{1} \sin ^{2 n-5} \theta_{2} \cdots \sin \theta_{n-1} \cos \theta_{1} \cos \theta_{2} \cdots \cos \theta_{n-1}$, and $J\left(\psi^{\prime}\right)=\sin ^{2 n-3} \theta_{1}^{\prime} \sin ^{2 n-5} \theta_{2}^{\prime} \cdots \sin \theta_{n-1}^{\prime} \cos \theta_{1}^{\prime} \cos \theta_{2}^{\prime} \cdots \cos \theta_{n-1}^{\prime}$.

Differentiating the composition of mappings

$$
\psi \longrightarrow u \longrightarrow v=g[u] \longrightarrow \psi^{\prime} \longrightarrow \widetilde{v}
$$

we get

$$
\frac{\partial \widetilde{v}}{\partial \psi}=\frac{\partial \widetilde{v}}{\partial \psi^{\prime}} \frac{\partial \psi^{\prime}}{\partial \psi}
$$

To verify (2), let us calculate $\operatorname{det}\left(\frac{\partial \widetilde{v}}{\partial \psi}\right)$ and $\operatorname{det}\left(\frac{\partial \widetilde{v}}{\partial \psi^{\prime}}\right)$.

As for the latter,

$$
\operatorname{det}\left(\frac{\partial \widetilde{v}}{\partial \psi^{\prime}}\right)=i^{n}(-2)^{n-1} J\left(\psi^{\prime}\right) v_{n}
$$


Then it is $\operatorname{det}\left(\frac{\partial \widetilde{v}}{\partial \psi}\right)$ 's turn.

$$
\begin{aligned}
\frac{\partial v_{j}}{\partial \theta_{k}} & =\frac{\left(\sum_{\ell=1}^{n} a_{j \ell} e^{i \varphi_{\ell}} t_{\ell, k}\right) \delta-\nu_{j}\left(\sum_{\ell=1}^{n} c_{\ell} e^{i \varphi_{\ell}} t_{\ell, k}\right)}{\delta^{2}} \\
& =\sum_{\ell=1}^{n} \alpha_{j \ell} e^{i \varphi_{\ell}} t_{\ell, k}, \quad\left(\alpha_{j \ell}=\frac{a_{j \ell} \delta-\nu_{j} c_{\ell}}{\delta^{2}}\right) \\
\frac{\partial u_{j}}{\partial \varphi_{k}} & =i \delta_{j k} u_{j}=i \delta_{j k} e^{i \varphi_{j}} t_{j}, \quad\left(\delta_{j k}: \text { Kronecker's delta }\right)^{\prime} \\
\frac{\partial u_{j}}{\partial \theta_{k}} & =e^{i \varphi_{j}} t_{j, k}, \quad\left(t_{j, k}=\frac{\partial t_{j}}{\partial \theta_{k}}\right) \\
v_{j}= & \frac{\nu_{j}}{\delta}, \quad\left(\begin{array}{l}
\left.\nu_{j}=\sum_{\ell=1}^{n} a_{j \ell} u_{\ell}+b_{j}, \quad \delta=\sum_{\ell=1}^{n} c_{\ell} u_{\ell}+d\right) \\
\frac{\partial v_{j}}{\partial \varphi_{k}}
\end{array}=\frac{i a_{j k} e^{i \varphi_{k}} t_{k} \delta-i \nu_{j} c_{k} e^{i \varphi_{k}} t_{k}}{\delta^{2}}=i \alpha_{j k} e^{i \varphi_{k}} t_{k}, \quad\left(\alpha_{j \ell}=\frac{a_{j \ell} \delta-\nu_{j} c_{\ell}}{\delta^{2}}\right)\right. \\
\frac{\partial \widetilde{v}}{\partial \psi} & =\left(\begin{array}{ll}
A B \\
C & D
\end{array}\right),
\end{aligned}
$$

where

$$
\begin{aligned}
& A=\left(i \alpha_{j k} e^{i \varphi_{k}} t_{k}\right) \in M_{n n}(\boldsymbol{C}), \quad B=\left(\sum_{\ell=1}^{n} \alpha_{j \ell} e^{i \varphi_{\ell}} t_{\ell, k}\right) \in M_{n n-1(\boldsymbol{C})}, \\
& C=\left(-i \overline{\alpha_{j k}} e^{-i \varphi_{k}} t_{k}\right) \in M_{n-1 n}(\boldsymbol{C}), \quad D=\left(\sum_{\ell=1}^{n} \overline{\alpha_{j \ell}} e^{-i \varphi_{\ell}} t_{\ell, k}\right) \in M_{n-1 n-1}(\boldsymbol{C}) .
\end{aligned}
$$

The determinant of left hand side of (3) is

$$
\begin{aligned}
& \operatorname{det}\left(\frac{\partial \widetilde{v}}{\partial \psi}\right) \\
& =i^{n} \operatorname{det}\left(\begin{array}{c}
\left(\alpha_{j k}\right)\left(\begin{array}{ccc}
e^{i \varphi_{1}} t_{1} & & \\
& \ddots & \\
& & e^{i \varphi_{n}} t_{n}
\end{array}\right) \\
\left(-\overline{\alpha_{j k}}\right)\left(\begin{array}{ccc}
e^{-i \varphi_{1}} t_{1} & & \\
& \ddots & \\
& & e^{-i \varphi_{n-1}} t_{n-1}
\end{array}\right) 2\left(\sum_{\ell=1}^{n} \overline{\alpha_{j \ell}} e^{-i \varphi_{\ell}} t_{\ell, k}\right)
\end{array}\right)
\end{aligned}
$$




$$
=i^{n} 2^{n-1} u_{1} \cdots u_{n} \operatorname{det}\left(\alpha_{j k}\right) \operatorname{det}\left(\sum_{\ell=1}^{n} \overline{\alpha_{j \ell}} e^{-i \varphi_{\ell}} t_{\ell, k}\right) .
$$

Using vectors $\alpha_{\ell}={ }^{t}\left(\alpha_{1 \ell}, \alpha_{2 \ell}, \ldots, \alpha_{n-1 \ell}\right) \in C^{n-1}(1 \leq l \leq n)$, we see that $\ell$-th column vector of the matrix $\left(\sum_{\ell=1}^{n} \overline{\alpha_{j \ell}} e^{-i \varphi_{\ell}} t_{\ell, k}\right)$ is of the form

$$
\overline{\alpha_{1}} e^{-i \varphi_{1}} t_{1, \ell}+\overline{\alpha_{2}} e^{-i \varphi_{2}} t_{2, \ell}+\cdots+\overline{\alpha_{n}} e^{-i \varphi_{n}} t_{n, \ell} .
$$

For each $\ell(1 \leq \ell \leq n)$, we put

$$
\lambda_{k}^{\ell}= \begin{cases}k & (k<\ell) \\ k+1 & (k \geq \ell)\end{cases}
$$

Now our calculation goes on. The last factor of the above equation is

$$
\begin{aligned}
& \operatorname{det}\left(\sum_{\ell=1}^{n} \overline{\alpha_{i \ell}} e^{-i \varphi_{\ell}} t_{\ell, k}\right) \\
& =\sum_{\ell=1}^{n} \operatorname{det}\left(\sum_{j=1}^{n-1} \overline{\alpha_{i \lambda_{j}^{\ell}}} e^{-i \varphi_{\lambda_{j}^{\ell}}} t_{\lambda_{j}^{\ell}, k}\right) \\
& =\sum_{\ell=1}^{n} \operatorname{det}\left(\overline{\alpha_{i \lambda_{k}^{\ell}}}\right) \operatorname{det}\left(e^{-i \varphi_{\lambda_{i}^{\ell}}} t_{\lambda_{i}^{\ell}, k}\right) \\
& =\sum_{\ell=1}^{n} \operatorname{det}\left(\overline{\alpha_{i \lambda_{k}^{\ell}}}\right) \operatorname{det}\left(t_{\lambda_{i}^{\ell}, k}\right) e^{-i\left(\varphi_{\lambda_{1}^{\ell}}+\varphi_{\lambda_{2}^{\ell}}+\cdots+\varphi_{\lambda_{n-1}^{\ell}}\right)} \\
& =\sum_{\ell=1}^{n}(-1)^{\ell-1} \operatorname{det}\left(\overline{\alpha_{i \lambda_{k}^{\ell}}}\right) s_{1} s_{2} \cdots s_{\ell} t_{\ell} s_{\ell+1} \cdots s_{n-1} e^{-i\left(\varphi_{\lambda_{1}^{\ell}}+\varphi_{\lambda_{2}^{\ell}}+\cdots+\varphi_{\lambda_{n-1}^{\ell}}\right)} .
\end{aligned}
$$

Therefore it follows

$$
\begin{aligned}
& u_{1} u_{2} \cdots u_{n} \operatorname{det}\left(\sum_{\ell=1}^{n} \overline{\alpha_{i \ell}} e^{-i \varphi_{\ell}} t_{\ell, k}\right) \\
& =\sum_{\ell=1}^{n}(-1)^{\ell-1} \operatorname{det}\left(\overline{\alpha_{i \lambda_{k}^{\ell}}}\right) s_{1} s_{2} \cdots s_{\ell} t_{\ell} s_{\ell+1} \cdots s_{n-1} t_{1} \cdots t_{\ell-1} u_{\ell} t_{\ell+1} \cdots t_{n} \\
& =\left(\sum_{\ell=1}^{n}(-1)^{\ell-1} \operatorname{det}\left(\overline{\alpha_{i \lambda_{k}^{\ell}}}\right) u_{\ell}\right) s_{2} \cdots s_{n-1} t_{1} \cdots t_{n} \\
& =(-1)^{n-1} \operatorname{det}\left(\begin{array}{cccc}
\overline{\alpha_{11}} & \overline{\alpha_{12}} & \cdots & \overline{\alpha_{1 n}} \\
\vdots & \vdots & \ddots & \vdots \\
\overline{\alpha_{n-11}} & \overline{\alpha_{n-12}} & \cdots & \overline{\alpha_{n-1 n}} \\
u_{1} & u_{2} & \cdots & u_{n}
\end{array}\right) t_{1} \cdots t_{n} s_{2} \cdots s_{n-1}
\end{aligned}
$$


so that

$$
\begin{aligned}
& \operatorname{det}\left(\frac{\partial \widetilde{v}}{\partial \psi}\right)
\end{aligned}
$$

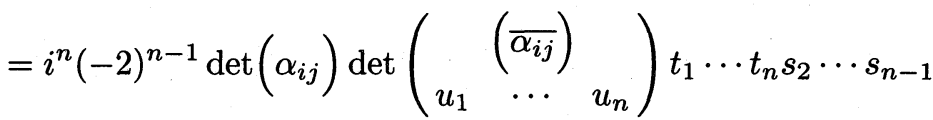

$$
\begin{aligned}
& =i^{n}(-2)^{n-1} t_{1} \cdots t_{n} s_{2} \cdots s_{n-1} \frac{\delta^{n-1}}{\delta^{2 n}} \frac{1}{(\bar{\delta})^{2(n-1)}} \operatorname{det}\left(\begin{array}{ccc}
\left(\overline{a_{i j} \delta-\nu_{i} c_{j}}\right. & \\
u_{1} & \cdots & u_{n}
\end{array}\right) \\
& =i^{n}(-2)^{n-1} J(\psi) \frac{1}{\delta^{n+1}} \frac{1}{(\bar{\delta})^{2 n-2}} \operatorname{det}\left(\begin{array}{cccc}
\overline{a_{11} \delta-\nu_{1} c_{1}} & \cdots & \overline{a_{1 n} \delta-\nu_{1} c_{n}} & 0 \\
\vdots & \ddots & \vdots & \vdots \\
u_{1} & \cdots & u_{n} & 0 \\
\overline{c_{1}} & \cdots & \overline{c_{n}} & 1
\end{array}\right) \\
& =i^{n}(-2)^{n-1} J(\psi) \frac{1}{\delta^{n+1}} \frac{1}{(\bar{\delta})^{2 n-2}} \operatorname{det}\left(\begin{array}{cccc}
\overline{a_{11} \delta} & \ldots & \overline{a_{1 n} \delta} & \overline{\nu_{1}} \\
\vdots & \ddots & \vdots & \vdots \\
\overline{a_{n-11} \delta} & \ldots & \overline{a_{n-1 n} \delta} & \overline{\nu_{n-1}} \\
u_{1} & \ldots & u_{n} & 0 \\
\overline{c_{1}} & \ldots & \overline{c_{n}} & 1
\end{array}\right) \\
& =i^{n}(-2)^{n-1} J(\psi) \frac{1}{\delta^{n+1}} \frac{(\bar{\delta})^{n-2}}{(\bar{\delta})^{2 n-2}} \operatorname{det}\left(\begin{array}{cccc}
\overline{a_{11}} & \cdots & \overline{a_{1 n}} & \overline{\nu_{1}} \\
\vdots & \ddots & \vdots & \vdots \\
\overline{a_{n-11}} & \cdots & \overline{a_{n-1 n}} & \overline{\nu_{n-1}} \\
u_{1} & \cdots & u_{n} & 0 \\
\overline{c_{1}} & \cdots & \overline{c_{n}} & \bar{\delta}
\end{array}\right) \\
& =\frac{i^{n}(-2)^{n-1}}{\delta|\delta|^{2 n}} J(\psi) \operatorname{det}\left(\begin{array}{cccc}
\overline{a_{11}} & \ldots & \overline{a_{1 n}} & \overline{b_{1}} \\
\vdots & \ddots & \vdots & \vdots \\
\overline{a_{n-11}} & \ldots & \overline{a_{n-1 n}} & \overline{b_{n-1}} \\
u_{1} & \ldots & u_{n} & -\left(u_{1} \overline{u_{1}}+\cdots+u_{n} \overline{u_{n}}\right) \\
\overline{c_{1}} & \ldots & \overline{c_{n}} & \bar{d}
\end{array}\right) \\
& =i^{n}(-2)^{n-1} J(\psi) \frac{1}{\delta^{n+1}} \frac{(\bar{\delta})^{n-2}}{(\bar{\delta})^{2 n-2}} \operatorname{det}\left(\begin{array}{cccc}
\overline{a_{11}} & \cdots & \overline{a_{1 n}} & \overline{\nu_{1}} \\
\vdots & \ddots & \vdots & \vdots \\
\overline{a_{n-11}} & \cdots & \overline{a_{n-1 n}} & \overline{\nu_{n-1}} \\
u_{1} & \cdots & u_{n} & 0 \\
\overline{c_{1}} & \ldots & \overline{c_{n}} & \bar{\delta}
\end{array}\right)
\end{aligned}
$$




$$
\begin{aligned}
& =\frac{i^{n}(-2)^{n-1}}{\delta|\delta|^{2 n}} J(\psi) \operatorname{det}\left(\begin{array}{cccc}
\overline{a_{11}} & \ldots & \overline{a_{1 n}} & \overline{b_{1}} \\
\vdots & \ddots & \vdots & \vdots \\
\overline{a_{n-11}} & \ldots & \overline{a_{n-1 n}} & \overline{b_{n-1}} \\
u_{1} & \ldots & u_{n} & -\left(u_{1} \overline{u_{1}}+\cdots+u_{n} \overline{u_{n}}\right) \\
\overline{c_{1}} & \ldots & \overline{c_{n}} & \bar{d}
\end{array}\right) \\
& =\frac{i^{n}(-2)^{n-1}}{|\delta|^{2 n}} J(\psi) \frac{1}{\delta} \operatorname{det}\left(\begin{array}{cccc}
\overline{a_{11}} & \cdots & \overline{a_{1 n}} & -\overline{b_{1}} \\
\vdots & \ddots & \vdots & \vdots \\
\overline{a_{n-11}} & \cdots & \overline{a_{n-1 n}} & -\overline{b_{n-1}} \\
u_{1} & \cdots & u_{n} & \frac{1}{d} \\
-\overline{c_{1}} & \cdots & -\overline{c_{n}} & \bar{d}
\end{array}\right) \\
& =\frac{i^{n}(-2)^{n-1}}{|c u+d|^{2 n}} J(\psi) v_{n} .
\end{aligned}
$$

This last equality follows from the above mentioned remark. Together with (4), this result brings us (2). Comparing the $(n+1, n+1)$-components of both sides of $g^{*} I_{n, 1} g=I_{n, 1}$ we have $d^{*} d-b^{*} b=1$. Since $\frac{b}{d}=z$ we get

$$
|d|^{2}=\frac{1}{1-z^{*} z}
$$

Replacing $g$ by $g^{-1}=\left(\begin{array}{cc}a^{*} & -c^{*} \\ -b^{*} & \bar{d}\end{array}\right)$, the formula (2) becomes

$$
\begin{aligned}
\frac{1}{\left|-b^{*} u+\bar{d}\right|^{2 n}} & =\frac{1}{\left|d-u^{*} b\right|^{2 n}}=\frac{1}{|d|^{2 n}} \frac{1}{\left|1-u^{*} b d^{-1}\right|} \\
& =\frac{\left(1-z^{*} z\right)^{n}}{\left|1-u^{*} z\right|^{2 n}}=\left(\frac{1-z^{*} z}{\left|1-u^{*} z\right|^{2}}\right)^{n}
\end{aligned}
$$

\section{Lie algebras}

We keep the notation of Section 1 . We assume that $G$ is simple and $P=M A N$ is maximal parabolic, so that $A$ is one dimensional. We denote by $\alpha$ the unique positive root on $\mathfrak{a}$. For any linear form $\lambda$ on $\mathfrak{a}$ we define

$$
\mathfrak{n}_{\lambda}=\{X \in \mathfrak{n} ;[H, X]=\lambda(H) X(X \in \mathfrak{n})\}
$$

Then the decomposition of $\mathfrak{n}$ into root spaces is given by

$$
\mathfrak{n}=\mathfrak{n}_{\alpha}+\mathfrak{n}_{2 \alpha}
$$


We choose an element $H$ in $\mathfrak{a}$ such that $\alpha(H)=1$. For simplicity we put

$$
\mathfrak{n}_{1}=\mathfrak{n}_{\alpha} . \quad \mathfrak{n}_{2}=\mathfrak{n}_{2 \alpha}
$$

We recall that $\rho(H)=\operatorname{Tr}\left(\left.a d(H)\right|_{\mathfrak{n}}\right)$. Then it is clear that $\rho(H)=\frac{1}{2}\left(\operatorname{dim} \mathfrak{n}_{1}+\right.$ $\left.2 \operatorname{dim} \mathfrak{n}_{2}\right)$.

\subsection{Type I}

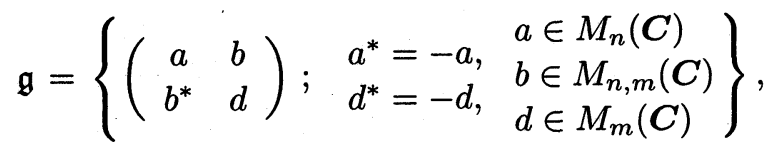

$\mathfrak{k}=\left\{\left(\begin{array}{ll}a & 0 \\ 0 & d\end{array}\right) ; \quad \begin{array}{ll}a^{*}=-a, & a \in M_{n}(\boldsymbol{C}) \\ d^{*}=-d, & d \in M_{m}(\boldsymbol{C})\end{array}\right\}$,

$\mathfrak{p}=\left\{\left(\begin{array}{cc}0 & b \\ b^{*} & 0\end{array}\right) ; b \in M_{n, m}(\boldsymbol{C})\right\}$,

$H=\left(\begin{array}{ccc}0 & 0 & I_{m} \\ 0 & 0 & 0 \\ I_{m} & 0 & 0\end{array}\right) \in M_{m+n}(\boldsymbol{R})$

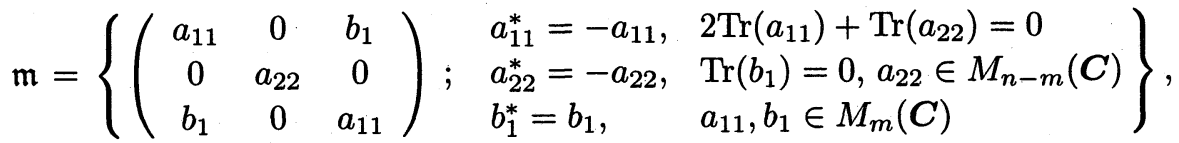

$\mathfrak{a}=\{t H ; t \in \boldsymbol{R}\}$,

$\mathfrak{n}=\left\{\left(\begin{array}{ccc}a & b & -a \\ -b^{*} & 0 & b^{*} \\ a & b & -a\end{array}\right) ; a^{*}=-a\left(a \in M_{m}(C), b \in M_{m, n-m}(C)\right)\right\}$,

$\mathfrak{n}_{1}=\left\{\left(\begin{array}{ccc}0 & b & 0 \\ -b^{*} & 0 & b^{*} \\ 0 & b & 0\end{array}\right) ; b \in M_{m, n-m}(C)\right\}$

$\mathfrak{n}_{2}=\left\{\left(\begin{array}{ccc}a & 0 & -a \\ 0 & 0 & 0 \\ a & 0 & -a\end{array}\right) ; a^{*}=-a, a \in M_{m}(C)\right\}$

$\mathfrak{g}_{c}=\mathfrak{s l}(m+n, C)$,

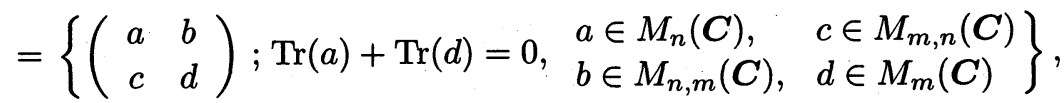




$$
\begin{aligned}
\mathfrak{k}_{c}= & \left\{\left(\begin{array}{ll}
a & 0 \\
0 & d
\end{array}\right) ; \operatorname{Tr}(a)+\operatorname{Tr}(d)=0\left(a \in M_{n}(\boldsymbol{C}), d \in M_{m}(\boldsymbol{C})\right)\right\} \\
\mathfrak{p}_{c}= & \left\{\left(\begin{array}{ll}
0 & b \\
c & 0
\end{array}\right) ; b \in M_{n, m}(\boldsymbol{C}), c \in M_{m, n}(\boldsymbol{C})\right\} \\
\mathfrak{p}_{+}= & \left\{\left(\begin{array}{ll}
0 & b \\
0 & 0
\end{array}\right) ; b \in M_{n, m}(\boldsymbol{C})\right\} \\
\mathfrak{p}_{-}= & \left\{\left(\begin{array}{ll}
0 & 0 \\
c & 0
\end{array}\right) ; c \in M_{m, n}(\boldsymbol{C})\right\} \\
& \operatorname{dim} \mathfrak{n}_{1}=2 m(n-m), \operatorname{dim} \mathfrak{n}_{2}=m^{2} \\
& \operatorname{dim} \mathfrak{n}_{1}+2 \operatorname{dim} \mathfrak{n}_{2}=2 m n
\end{aligned}
$$

\subsection{Type II}

$$
\begin{aligned}
& \mathfrak{g}=\left\{\left(\begin{array}{cc}
a & b \\
\bar{b} & \bar{a}
\end{array}\right) ; a^{*}=-a,{ }^{t} b=b\left(a, b \in M_{n}(C)\right)\right\} \\
& \mathfrak{k}=\left\{\left(\begin{array}{cc}
a & 0 \\
0 & \bar{a}
\end{array}\right) ; a^{*}=-a\left(a \in M_{n}(C)\right)\right\}, \\
& \mathfrak{p}=\left\{\left(\begin{array}{cc}
0 & b \\
\bar{b} & 0
\end{array}\right) ;{ }^{t} b=b\left(b \in M_{n}(\boldsymbol{C})\right)\right\}, \\
& H=\left(\begin{array}{cc}
0 & I_{n} \\
I_{n} & 0
\end{array}\right) \\
& \mathfrak{m}=\left\{\left(\begin{array}{cc}
a & b \\
b & a
\end{array}\right) ;{ }^{t} a=-a,{ }^{t} b=b, \operatorname{Tr}(b)=0\left(a, b \in M_{n}(\boldsymbol{R})\right)\right\} \\
& \mathfrak{a}=\{t H ; t \in \boldsymbol{R}\} \\
& \mathfrak{n}=\left\{\left(\begin{array}{cc}
i a & -i a \\
i a & -i a
\end{array}\right) ;{ }^{t} a=a\left(a \in M_{n}(\boldsymbol{R})\right)\right\} \\
& \mathfrak{n}_{1}=\{0\}, \\
& \mathfrak{n}_{2}=\mathfrak{n}, \\
& \mathfrak{g}_{c}=\mathfrak{s p}(n, C), \\
& =\left\{\left(\begin{array}{cc}
a & b \\
c & -{ }^{t} a
\end{array}\right) ;{ }^{t} b=b,{ }^{t} c=c\left(a, b, c \in M_{n}(\boldsymbol{C})\right)\right\}, \\
& \mathfrak{k}_{c}=\left\{\left(\begin{array}{cc}
a & 0 \\
0 & -{ }^{t} a
\end{array}\right) ; a \in M_{n}(\boldsymbol{C})\right\},
\end{aligned}
$$




$$
\begin{aligned}
\mathfrak{p}_{c}= & \left\{\left(\begin{array}{ll}
0 & b \\
c & 0
\end{array}\right) ;{ }^{t} b=b,{ }^{t} c=c\left(b, c \in M_{n}(C)\right)\right\}, \\
\mathfrak{p}_{+}= & \left\{\left(\begin{array}{ll}
0 & b \\
0 & 0
\end{array}\right) ;{ }^{t} b=b\left(b \in M_{n}(C)\right)\right\}, \\
\mathfrak{p}_{-}= & \left\{\left(\begin{array}{ll}
0 & 0 \\
c & 0
\end{array}\right) ;{ }^{t} c=c\left(c \in M_{n}(C)\right)\right\} . \\
& \operatorname{dim} \mathfrak{n}_{1}=0, \operatorname{dim} \mathfrak{n}_{2}=\frac{n(n+1)}{2}, \\
& \operatorname{dim} \mathfrak{n}_{1}+2 \operatorname{dim} \mathfrak{n}_{2}=n(n+1) .
\end{aligned}
$$

\subsection{Type III}

Fix any $n(n>1) \in \boldsymbol{N}$. Define

$$
\begin{aligned}
& \mathfrak{g}=\left\{\left(\begin{array}{cc}
a & b \\
-\bar{b} & \bar{a}
\end{array}\right) ; a^{*}=-a,{ }^{t} b=-b\left(a, b \in M_{n}(\boldsymbol{C})\right)\right\} \\
& \mathfrak{k}=\left\{\left(\begin{array}{cc}
a & 0 \\
0 & \bar{a}
\end{array}\right) ; a^{*}=-a\left(a \in M_{n}(\boldsymbol{C})\right)\right\}, \\
& \mathfrak{p}=\left\{\left(\begin{array}{cc}
0 & b \\
-\bar{b} & 0
\end{array}\right) ;{ }^{t} b=-b\left(b \in M_{n}(\boldsymbol{C})\right)\right\}, \\
& \mathfrak{g}_{c}=\left\{\left(\begin{array}{cc}
a & b \\
c & -{ }^{t} a
\end{array}\right) ; \quad \begin{array}{l}
{ }^{t} b=-b, \\
{ }^{t} c=-c
\end{array}\left(a, b, c \in M_{n}(\boldsymbol{C})\right)\right\}, \\
& \mathfrak{k}_{c}=\left\{\left(\begin{array}{cc}
a & 0 \\
0 & -{ }^{t} a
\end{array}\right) ; a \in M_{n}(C)\right\} \\
& \mathfrak{p}_{c}=\left\{\left(\begin{array}{cc}
0 & b \\
c & 0
\end{array}\right) ; \quad \begin{array}{ll}
{ }^{t} b=-b, & b \in M_{n}(\boldsymbol{C}) \\
t^{t} c=-c, & c \in M_{n}(\boldsymbol{C})
\end{array}\right\}, \\
& \mathfrak{p}_{+}=\left\{\left(\begin{array}{cc}
0 & b \\
0 & 0
\end{array}\right) ;{ }^{t} b=-b\left(b \in M_{n}(\boldsymbol{C})\right)\right\}, \\
& \mathfrak{p}_{-}=\left\{\left(\begin{array}{cc}
0 & 0 \\
c & 0
\end{array}\right) ;{ }^{t} c=-c\left(c \in M_{n}(\boldsymbol{C})\right)\right\} \text {. }
\end{aligned}
$$

\subsubsection{Case n: even}

Fix any $n \in N$. Put $m=\frac{n}{2}$. Define

$$
\sigma=\left(\begin{array}{cc}
0 & I_{m} \\
-I_{m} & 0
\end{array}\right)
$$




$$
\begin{aligned}
& H=\left(\begin{array}{cc}
0 & \sigma \\
-\sigma & 0
\end{array}\right) \\
& \mathfrak{m}=\left\{\left(\begin{array}{cc}
a & b \\
-\bar{b} & \bar{a}
\end{array}\right) \in \mathfrak{s l}(2 n, C) ; a, b \text { satisfy the condition }(*) \text { bellow }\right\}, \\
& (*) \begin{cases}a= & \left.\begin{array}{cc}
a_{11} & a_{12} \\
-\overline{a_{12}} & \overline{a_{11}}
\end{array}\right) \begin{array}{l}
a_{11}=-a_{11}^{*}, \\
a_{12}={ }^{t} a_{12},
\end{array} \quad\left(a_{11}, a_{12} \in M_{\frac{n}{2}}(\boldsymbol{C})\right), \\
b=\left(\begin{array}{cc}
b_{11} & b_{12} \\
-\overline{b_{12}} & \overline{b_{11}}
\end{array}\right) \begin{array}{l}
b_{11}=-{ }^{t} b_{11}, \\
b_{12}=b_{12}^{*}
\end{array} \quad\left(b_{11}, b_{12} \in M_{\frac{n}{2}}(\boldsymbol{C})\right) .\end{cases} \\
& \mathfrak{a}=\{t H ; t \in \boldsymbol{R}\}, \\
& \mathfrak{n}=\left\{\left(\begin{array}{cc}
a & b \\
-\bar{b} & \bar{a}
\end{array}\right) ; a, b \text { satisfy the condition }(*) \text { bellow }\right\}, \\
& (*)\left\{\begin{array}{l}
a=\left(\begin{array}{cc}
a_{11} & a_{12} \\
\overline{a_{12}} & -\overline{a_{11}}
\end{array}\right), \\
b=\left(\begin{array}{cc}
a_{12} & -a_{11} \\
-\overline{a_{11}} & -\overline{a_{12}}
\end{array}\right), \\
a_{11}=-a_{11}^{*}, \quad\left(a_{11}, a_{12} \in M_{\frac{n}{2}}\right) . \\
a_{12}=-{ }^{t} a_{12}
\end{array}\right. \\
& \mathfrak{n}_{1}=\{0\}, \\
& \mathfrak{n}_{2}=\mathfrak{n} \text {. } \\
& \operatorname{dim} \mathfrak{n}_{1}=0, \operatorname{dim} \mathfrak{n}_{2}=\frac{n(n-1)}{2}, \\
& \operatorname{dim} \mathfrak{n}_{1}+2 \operatorname{dim} \mathfrak{n}_{2}=n(n-1) .
\end{aligned}
$$

\subsubsection{Case n: odd}

Fix any $n \in N$. Put $m=\frac{n-1}{2}$. Define

$$
\begin{aligned}
\sigma & =\left(\begin{array}{ccc}
0 & 0 & I_{m} \\
0 & 0 & 0 \\
-I_{m} & 0 & 0
\end{array}\right), \\
H & =\left(\begin{array}{cc}
0 & \sigma \\
-\sigma & 0
\end{array}\right), \\
\mathfrak{m} & =\left\{\left(\begin{array}{cc}
a & b \\
-\bar{b} & \bar{a}
\end{array}\right) \in \mathfrak{s l}(2 n, \boldsymbol{C}) \begin{array}{ll}
a^{*}=-a, & \sigma \bar{a}=a \sigma, \\
t b=-b, & \sigma \bar{b}=b \sigma, \\
\mathfrak{a} & =\{t H ; t \in \boldsymbol{R}\},
\end{array}\right.
\end{aligned}
$$


$\mathfrak{n}=\left\{\left(\begin{array}{cc}a & b \\ -\bar{b} & \bar{a}\end{array}\right) ; a, b\right.$ satisfy the condition $(*)$ bellow $\}$

$$
(*)\left\{\begin{array}{l}
a=\left(\begin{array}{ccc}
a_{11} & a_{12} & a_{13} \\
-a_{12}^{*} & 0 & a_{23} \\
\overline{a_{13}} & -a_{23}^{*} & -\overline{a_{11}}
\end{array}\right), \\
b=\left(\begin{array}{ccc}
a_{13} & -{ }^{t} a_{23} & -a_{11} \\
a_{23} & 0 & a_{12}^{*} \\
-\overline{a_{11}} & -\overline{a_{12}} & -\overline{a_{13}}
\end{array}\right), \\
a_{11}=-a_{11}^{*}, a_{13}=-{ }^{t} a_{13}\left(a_{11}, a_{13} \in M_{m}(C), a_{12} \in M_{m, 1}, a_{23} \in M_{1, m}\right) .
\end{array}\right.
$$

$\mathfrak{n}_{1}=\left\{\left(\begin{array}{cc}a & b \\ -\bar{b} & \bar{a}\end{array}\right) ; a, b\right.$ satisfy the condition (*) bellow $\}$,

$$
\begin{aligned}
& \text { (*) }\left\{\begin{array}{c}
a=\left(\begin{array}{ccc}
0 & a_{12} & 0 \\
-a_{12}^{*} & 0 & a_{23} \\
0 & -a_{23}^{*} & 0
\end{array}\right), \\
b=\left(\begin{array}{ccc}
0 & -{ }^{t} a_{23} & 0 \\
a_{23} & 0 & a_{12}^{*} \\
0 & -\overline{a_{12}} & 0
\end{array}\right), \\
\left(a_{12} \in M_{m, 1}(C), a_{23} \in M_{1, m}(C)\right) .
\end{array}\right. \\
& \mathfrak{n}_{2}=\left\{\left(\begin{array}{cc}
a & b \\
-\bar{b} & \bar{a}
\end{array}\right) ; a, b \text { satisfy the condition }(*) \text { bellow }\right\} \text {, } \\
& (*)\left\{\begin{array}{l}
a=\left(\begin{array}{ccc}
a_{11} & 0 & a_{13} \\
0 & 0 & 0 \\
\overline{a_{13}} & 0 & -\overline{a_{11}}
\end{array}\right), \\
b=\left(\begin{array}{ccc}
a_{13} & 0 & -a_{11} \\
0 & 0 & 0 \\
-\overline{a_{11}} & 0 & -\overline{a_{13}}
\end{array}\right), \\
a_{11}=-a_{11}^{*}, a_{13}=-{ }^{t} a_{13}\left(a_{11}, a_{13} \in M_{m}\right) .
\end{array}\right. \\
& \operatorname{dim} \mathfrak{n}_{1}=2(n-1), \operatorname{dim} \mathfrak{n}_{2}=\frac{(n-1)(n-2)}{2}, \\
& \operatorname{dim} \mathfrak{n}_{1}+2 \operatorname{dim} \mathfrak{n}_{2}=n(n-1) \text {. }
\end{aligned}
$$

\subsection{Type IV}

$$
\mathfrak{g}=\left\{\left(\begin{array}{cc}
a & b \\
{ }^{t} b & d
\end{array}\right) ; \quad \begin{array}{c}
{ }^{t} a=-a, \\
{ }^{t} d=-d
\end{array}\left(a \in M_{n}(\boldsymbol{R}), b \in M_{n, 2}(\boldsymbol{R}), d \in M_{2}(\boldsymbol{R})\right)\right\}
$$




$$
\begin{aligned}
& \mathfrak{k}=\left\{\left(\begin{array}{cc}
a & 0 \\
0 & d
\end{array}\right) ; \quad \begin{array}{l}
{ }^{t} a=-a, \\
{ }^{t} d=-d
\end{array}\left(a \in M_{n}(\boldsymbol{R}), d \in M_{2}(\boldsymbol{R})\right)\right\} \\
& \mathfrak{p}=\left\{\left(\begin{array}{cc}
0 & b \\
{ }^{t} b & 0
\end{array}\right) ; b \in M_{n, 2}(R)\right\} \\
& H=\left(\begin{array}{lll}
0 & 0 & 1 \\
0 & 0 & 0 \\
1 & 0 & 0
\end{array}\right) \in M_{n+2}(R), \\
& \mathfrak{m}=\left\{\left(\begin{array}{cccc}
0 & 0 & 0 & 0 \\
0 & m_{11} & m_{12} & 0 \\
0 & { }^{t} m_{12} & 0 & 0 \\
0 & 0 & 0 & 0
\end{array}\right) ;{ }^{t} m_{11}=-m_{11},\left(m_{11} \in M_{n-1}(R), m_{12} \in R^{n-1}\right)\right\}, \\
& \mathfrak{a}=\{t H ; t \in \boldsymbol{R}\}, \\
& \mathfrak{n}=\left\{\left(\begin{array}{cccc}
0 & a & b & 0 \\
-{ }^{t} a & 0 & 0 & { }^{t} a \\
b & 0 & 0 & -b \\
0 & a & b & 0
\end{array}\right) \in M_{n+2}(\boldsymbol{R}) ;{ }^{t} a \in \boldsymbol{R}^{n-1}, b \in \boldsymbol{R}\right\} \\
& \mathfrak{n}_{1}=\mathfrak{n}, \\
& \mathfrak{n}_{2}=\{0\} \\
& \mathfrak{g}_{c}=\mathfrak{o}(n+2, C) \text {, } \\
& =\left\{\left(\begin{array}{cc}
a & b \\
-{ }^{t} b & d
\end{array}\right) ;{ }^{t} a=-a,{ }^{t} d=-d\left(a \in M_{n}(\boldsymbol{C}), d \in M_{2}(\boldsymbol{C})\right)\right\}, \\
& \mathfrak{k}_{c}=\left\{\left(\begin{array}{ll}
a & 0 \\
0 & d
\end{array}\right) ;{ }^{t} a=-a,{ }^{t} d=-d\left(a \in M_{n}(C), d \in M_{2}(C)\right)\right\} .
\end{aligned}
$$

Put

$$
\begin{aligned}
& \gamma=\left(\begin{array}{cc}
I_{n} & 0 \\
0 & \left(\begin{array}{cc}
i & 1 \\
-i & 1
\end{array}\right)
\end{array}\right) \\
& \tilde{\mathfrak{g}}_{c}=\gamma \mathfrak{g}_{c} \gamma^{-1} \text {. }
\end{aligned}
$$

Define

$$
\tilde{\mathfrak{p}}_{c}=\left\{\left(\begin{array}{ccc}
0 & w & z \\
-2{ }^{t} z & 0 & 0 \\
-2^{t} w & 0 & 0
\end{array}\right) \in \tilde{\mathfrak{g}}_{c} ; z, w \in C^{n}\right\}
$$




$$
\begin{aligned}
& \widetilde{\mathfrak{p}}_{+}=\left\{\left(\begin{array}{ccc}
0 & 0 & z \\
-2{ }^{t} z & 0 & 0 \\
0 & 0 & 0
\end{array}\right) \in \widetilde{\mathfrak{g}}_{c} ; z \in C^{n}\right\}, \\
& \tilde{\mathfrak{p}}_{-}=\left\{\left(\begin{array}{ccc}
0 & w & 0 \\
0 & 0 & 0 \\
-2{ }^{t} w & 0 & 0
\end{array}\right) \in \tilde{\mathfrak{g}}_{c} ; w \in C^{n}\right\} .
\end{aligned}
$$

Then

$$
\begin{aligned}
\mathfrak{p}_{+}= & \gamma^{-1} \widetilde{\mathfrak{p}}_{+} \gamma \\
\mathfrak{p}_{-}= & \gamma^{-1} \tilde{\mathfrak{p}}_{-} \gamma \\
& \operatorname{dim} \mathfrak{n}_{1}=n, \operatorname{dim} \mathfrak{n}_{2}=0 \\
& \operatorname{dim} \mathfrak{n}_{1}+2 \operatorname{dim} \mathfrak{n}_{2}=n
\end{aligned}
$$

\section{Generalized Poisson and Cauchy transformations}

We keep the notation of Section 1. Let $\tau$ be a holomorphic character of $K$. Then $\tau$ is uniquely extended to a holomorphic character of $U$ which is trivial on $P_{-}$. We regard the complex Lie group $G_{c}$ as the principal fiber bundle over the complex homogeneous space $G_{c} / U$. For any holomorphic character $\tau$ of $U$ we denote by $\widetilde{E}_{\tau}$ the holomorphic line bundle over $G_{c} / U$ associated to $\tau$. Then the space of all $C^{\infty}$-sections of $\widetilde{E}_{\tau}$ is identified with

$$
\boldsymbol{C}^{\infty}\left(\widetilde{E}_{\tau}\right)=\left\{h \in \boldsymbol{C}^{\infty}\left(G_{c}\right) ; h(w u)=\tau(u)^{-1} h(w) \quad\left(w \in G_{c}, u \in U\right)\right\}
$$

We denote by $E_{\tau}$ the restriction of $\widetilde{E}_{\tau}$ to the open submanifold $G / K \cong G U / U$ of $G_{c} / U$. Then the space of all $C^{\infty}$-sections of $E_{\tau}$ is identified with

$$
C^{\infty}\left(E_{\tau}\right)=\left\{h \in C^{\infty}(G U) ; h(w u)=\tau(u)^{-1} h(w) \quad(w \in G U, u \in U)\right\} .
$$

Let $\eta$ be a $C^{\infty}$-character of $U$ such that the restriction of $\eta$ to $\mathrm{K}$ coincides with the restriction of $\tau$ to $\mathrm{K}$. We denote by $\widetilde{L}_{\eta}$ the $C^{\infty}$-line bundle on $G_{c} / U$ associated to $\eta$. Then the space of all $C^{\infty}$-sections of $\widetilde{L}_{\eta}$ is identified with

$$
\boldsymbol{C}^{\infty}\left(\widetilde{L}_{\eta}\right)=\left\{h \in \boldsymbol{C}^{\infty}\left(G_{c}\right) ; h(w u)=\eta(u)^{-1} h(w) \quad\left(w \in G_{c}, u \in U\right)\right\} .
$$

We denote by $L_{\eta}$ the restriction of $\widetilde{L}_{\eta}$ to the compact submanifold $G / P \cong$ $G \mu_{o} U / U$ of $G_{c} / U$. Then the space of all $C^{\infty}$-sections of $L_{\eta}$ is identified with

$$
\boldsymbol{C}^{\infty}\left(L_{\eta}\right)=\left\{h \in \boldsymbol{C}^{\infty}\left(G \mu_{o} U\right) ; h(w u)=\eta(u)^{-1} h(w) \quad\left(w \in G \mu_{o} U, u \in U\right)\right\} .
$$


The canonical onto-isomorphisms:

$$
G / K \cong G U / U \cong D \text { and } G / P \cong G \mu_{o} U / U \cong \check{S}
$$

are given by

$$
g K \longmapsto g U \longmapsto g[0]=z \text { and } g P \longmapsto g \mu_{o} U \longmapsto g\left[u_{o}\right]=u,
$$

respectively. We define the $C^{\infty}$-character $\xi$ of $P$ by

$$
\xi(p)=\eta\left(\mu_{o}^{-1} p \mu_{o}\right) \quad(p \in P) .
$$

Put

$$
\begin{aligned}
& C^{\infty}(G)_{\tau}=\left\{f \in C^{\infty}(G) ; f(g k)=\tau(k)^{-1} f(g) \quad(g \in G, k \in K)\right\} \\
& C^{\infty}(G)_{\xi}=\left\{\phi \in C^{\infty}(G) ; \phi(g p)=\xi(p)^{-1} \phi(g) \quad(g \in G, p \in P)\right\}
\end{aligned}
$$

Then we obtain the following four onto-isomorphisms:

$$
\begin{aligned}
& C^{\infty}\left(E_{\tau}\right) \ni h \longmapsto f \in C^{\infty}(G)_{\tau}, \quad f(g)=h(g) \quad(g \in G), \\
& C^{\infty}\left(E_{\tau}\right) \ni h \longmapsto F \in C^{\infty}(D), \quad F(z)=h(\exp z) \quad(z \in \boldsymbol{D}), \\
& C^{\infty}\left(L_{\eta}\right) \ni \psi \longmapsto \phi \in C^{\infty}(G)_{\xi}, \quad \phi(g)=\psi\left(g \mu_{o}\right) \quad(g \in G), \\
& C^{\infty}\left(L_{\eta}\right) \ni \psi \longmapsto \Phi \in C^{\infty}(\check{\boldsymbol{S}}), \quad \Phi(u)=\psi(\exp u) \quad(u \in \check{\boldsymbol{S}}) .
\end{aligned}
$$

- Now, we define the generalized Poisson-Cauchy transform

$$
\mathcal{P}_{\tau, \eta}: C^{\infty}(G)_{\xi} \ni \phi \longmapsto f \in C^{\infty}(G)_{\tau}
$$

by

$$
f(g)=\int_{K} \tau(k) \phi(g k) d k \quad(g \in G)
$$

\subsection{The simplest example}

We start with the simplest example.

$$
\begin{aligned}
& G=S U(1,1) \cong S L(2, \boldsymbol{R}), \\
& S U(1,1)=\left\{\left(\begin{array}{cc}
a & b \\
\bar{b} & \bar{a}
\end{array}\right) ;|a|^{2}-|b|^{2}=1\right\} \\
& k_{\theta}=\left(\begin{array}{cc}
e^{i \theta / 2} & 0 \\
0 & e^{-i \theta / 2}
\end{array}\right), \quad a_{t}=\left(\begin{array}{cc}
\operatorname{ch} t & \operatorname{sh} t \\
\operatorname{sh} t & \operatorname{ch} t
\end{array}\right), \quad n_{x}=\left(\begin{array}{cc}
1+i x & -i x \\
i x & 1-i x
\end{array}\right),
\end{aligned}
$$




$$
\begin{aligned}
& K=\left\{k_{\theta} ; 0 \leq \theta<4 \pi\right\}, A=\left\{a_{t} ; t \in \boldsymbol{R}\right\}, \\
& M=\left\{\left(\begin{array}{cc}
1 & 0 \\
0 & 1
\end{array}\right),\left(\begin{array}{cc}
-1 & 0 \\
0 & -1
\end{array}\right)\right\}, \quad N=\left\{n_{x} ; x \in \boldsymbol{R}\right\}, \\
& G=K A N, \quad P=M A N, \\
& G_{c}=S L(2, C) \text {, } \\
& K_{c}=\left\{\left(\begin{array}{cc}
\alpha & 0 \\
0 & \alpha^{-1}
\end{array}\right) ; \alpha \in C^{*}\right\} \text {, } \\
& P_{+}=\left\{\left(\begin{array}{cc}
1 & z \\
0 & 1
\end{array}\right) ; z \in C\right\} \\
& P_{-}=\left\{\left(\begin{array}{cc}
1 & 0 \\
\zeta & 1
\end{array}\right) ; \zeta \in C\right\} \\
& B=K_{c} P_{-} \text {, } \\
& G \cap \mu B \mu^{-1}=G \cap \mu_{o} B \mu_{o}^{-1}=P, \quad \mu=\frac{1}{1+i}\left(\begin{array}{cc}
i & 1 \\
-i & 1
\end{array}\right), \quad \mu_{o}=\left(\begin{array}{ll}
1 & 1 \\
0 & 1
\end{array}\right), \\
& \boldsymbol{D}=\{z \in \boldsymbol{C} ;|z|<1\} \cong G B / B \cong G / K, \\
& \check{\boldsymbol{S}}=\{z \in C ;|z|=1\}=\left\{e^{i \theta} ; 0 \leq \theta<2 \pi\right\} \cong G \mu B / B \cong G \mu_{o} B / B \cong G / P, \\
& C^{\infty}\left(E_{n}\right)=\left\{f \in C^{\infty}(G B) ; f(w b)=\tau_{n}(b)^{-1} f(w) \quad(w \in G B, b \in B)\right\}, \\
& C^{\infty}(G)_{n}=\left\{f \in C^{\infty}(G) ; f(g k)=\tau_{n}(k)^{-1} f(g) \quad(g \in G, k \in K)\right\}, \\
& C^{\infty}\left(L_{n, s}\right)=\left\{f \in C^{\infty}\left(G \mu_{o} B\right)_{n, s} ; f(w b)=\eta_{n, s}(b)^{-1} f(w) \quad\left(w \in G \mu_{o} B, b \in B\right)\right\}, \\
& C^{\infty}(G)_{n, s}=\left\{f \in C^{\infty}(G) ; f(g p)=\xi_{n, s}(p)^{-1} f(g) \quad(g \in G, p \in P)\right\} .
\end{aligned}
$$

In the above, for any $n \in N, s \in C, b=\left(\begin{array}{cc}\alpha & 0 \\ \zeta & \alpha^{-1}\end{array}\right) \in B$ and $p \in P$, we put $\tau_{n}(b)=\alpha^{-n}, \quad \eta_{n, s}(b)=\left(\frac{\alpha}{|\alpha|}\right)^{-n}|\alpha|^{-s} \quad$ and $\quad \xi_{n, s}(p)=\eta_{n, s}\left(\mu_{o}^{-1} p \mu_{o}\right)$.

Then we have

$$
\begin{aligned}
\tau_{n}\left(k_{\theta}\right) & =e^{-i n \theta / 2} \\
\xi_{n, s}\left(a_{t} n_{x}\right) & =\eta_{n, s}\left(\mu_{o}^{-1} a_{t} n_{x} \mu_{o}\right) \\
& =\eta_{n, s}\left(\left(\begin{array}{cc}
e^{-t} & 0 \\
\operatorname{sh} t+i e^{t} x & e^{t}
\end{array}\right)\right) \\
& =e^{s t} .
\end{aligned}
$$


For any $g=\left(\begin{array}{cc}a & b \\ \bar{b} & \bar{a}\end{array}\right)$, put $z=\frac{b}{\bar{a}}$. Then since $|a|^{2}-|b|^{2}=1$, we have

$$
1-|z|^{2}=1-\left|\frac{b}{\bar{a}}\right|^{2}=|a|^{-2}>0 .
$$

For any $g=\left(\begin{array}{cc}a & b \\ \bar{b} & \bar{a}\end{array}\right)$, put $e^{i \theta}=\frac{a+b}{\bar{a}+\bar{b}}$. Then we have

$$
\begin{aligned}
g=\left(\begin{array}{ll}
a & b \\
\bar{b} & \bar{a}
\end{array}\right)=\left(\begin{array}{ll}
1 & \frac{b}{\bar{a}} \\
0 & 1
\end{array}\right)\left(\begin{array}{cc}
\frac{1}{\bar{a}} & 0 \\
\bar{b} & \bar{a}
\end{array}\right) & \equiv\left(\begin{array}{cc}
1 & z \\
0 & 1
\end{array}\right) \quad(\bmod B) \\
g \mu_{o}=\left(\begin{array}{ll}
a & b \\
\bar{b} & \bar{a}
\end{array}\right)\left(\begin{array}{ll}
1 & 1 \\
0 & 1
\end{array}\right)=\left(\begin{array}{cc}
1 & \frac{a+b}{\bar{a}+\bar{b}} \\
0 & 1
\end{array}\right) & \left(\begin{array}{cc}
\frac{1}{\bar{a}+\bar{b}} & 0 \\
\bar{b} & \bar{a}+\bar{b}
\end{array}\right) \\
& \equiv\left(\begin{array}{cc}
1 & e^{i \theta} \\
0 & 1
\end{array}\right) \quad(\bmod B) .
\end{aligned}
$$

Moreover, the above onto-isomorphisms:

$G / K \cong G B / B \cong D$ and $G / P \cong G \mu_{o} B / B \cong \check{S}$ are given by

$$
\begin{aligned}
& g K \longmapsto\left(\begin{array}{cc}
1 & \frac{b}{\bar{a}} \\
0 & 1
\end{array}\right) B \longmapsto \frac{b}{\bar{a}}=z, \\
& g P \longmapsto\left(\begin{array}{cc}
1 & \frac{a+b}{\bar{a}+\bar{b}} \\
0 & 1
\end{array}\right) B \longmapsto \frac{a+b}{\bar{a}+\bar{b}}=e^{i \theta} .
\end{aligned}
$$

The canonical action of $G$ on $G / K$ induces the action on $D$ by the following commutative diagrams.

$$
\begin{aligned}
& \text { For } g_{1}=\left(\begin{array}{ll}
a_{1} & b_{1} \\
\overline{b_{1}} & \overline{a_{1}}
\end{array}\right) \\
& \begin{array}{ccc}
G / K \ni g K & \longmapsto & g_{1} g K \in G / K \\
\downarrow & \downarrow & \downarrow \\
D \ni z & \longmapsto & \frac{a_{1} z+b_{1}}{\overline{b_{1}} z+\overline{a_{1}}} \in \boldsymbol{D} .
\end{array}
\end{aligned}
$$

Furthermore, the canonical action of $G$ on $G / P$ induces the action on $\check{\boldsymbol{S}}$ by 
the following commutative diagrams. For $g_{1}=\left(\begin{array}{cc}a_{1} & b_{1} \\ \overline{b_{1}} & \frac{a_{1}}{}\end{array}\right)$

$$
\begin{array}{ccc}
G / P \ni g P & \longmapsto & g_{1} g P \in G / P \\
\downarrow & \downarrow & \downarrow \\
\check{\boldsymbol{S}} \ni e^{i \theta} & \longmapsto & \frac{a_{1} e^{i \theta}+b_{1}}{\overline{b_{1}} e^{i \theta}+\overline{a_{1}}} \in \check{\boldsymbol{S}}
\end{array}
$$

Using these onto-isomorphisms, we obtain the following four onto-isomorphisms:

$$
\begin{aligned}
& C^{\infty}\left(E_{n}\right) \ni h \longmapsto f \in C^{\infty}(G)_{n}, \quad f(g)=h(g) \quad(g \in G), \\
& C^{\infty}\left(E_{n}\right) \ni h \longmapsto F \in C^{\infty}(D), \quad F(z)=h\left(\left(\begin{array}{cc}
1 & z \\
0 & 1
\end{array}\right)\right) \quad(z \in D), \\
& C^{\infty}\left(L_{n, s}\right) \ni \psi \longmapsto \phi \in C^{\infty}(G)_{n, s}, \quad \phi(g)=\psi\left(g \mu_{o}\right) \quad(g \in G), \\
& C^{\infty}\left(L_{n, s}\right) \ni \psi \longmapsto \Phi \in C^{\infty}(S), \quad \Phi\left(e^{i \theta}\right)=\psi\left(\left(\begin{array}{cc}
1 & e^{i \theta} \\
0 & 1
\end{array}\right)\right) \quad\left(e^{i \theta} \in \check{S}\right) .
\end{aligned}
$$

Now, we define the map

$$
\mathcal{P}_{n, s}: C^{\infty}(G)_{n, s} \ni \phi \longmapsto f \in C^{\infty}(G)_{n}
$$

by

$$
f(g)=\int_{K} \tau_{n}(k) \phi(g k) d k=\frac{1}{4 \pi} \int_{0}^{4 \pi} \tau_{n}\left(k_{\theta}\right) \phi\left(g k_{\theta}\right) d \theta \quad(g \in G) .
$$

In the following we prove that this mapping $\mathcal{P}_{n, s}$ gives us the generalized Poisson and Cauchy kernel functions.

Let $f=\mathcal{P}_{n, s}(\phi)$. Then for any $g \in G$ we have

$$
f(g)=\frac{1}{4 \pi} \int_{0}^{4 \pi} \tau_{n}\left(k_{\varphi}\right) \phi\left(g k_{\varphi}\right) d \varphi
$$

For $g=\left(\begin{array}{cc}a & b \\ \bar{b} & \bar{a}\end{array}\right)$, we put

$$
g k_{\varphi}=k_{\theta} a_{t} n_{x}
$$

Then we have

$$
g^{-1} k_{\theta}=k_{\varphi} a_{-t} n_{-e^{2 t} x}
$$


Applying both sides of this equation to the vector $\left(\begin{array}{l}1 \\ 1\end{array}\right)$ and comparing the second components, we obtain

$$
e^{-i \varphi / 2} e^{-t}=-\bar{b} e^{i \theta / 2}+a e^{-i \theta / 2}
$$

Since $|a|^{2}-|b|^{2}=1$ and $\frac{b}{\bar{a}}=z$, it follows that

$$
\begin{aligned}
\tau_{n}\left(k_{\varphi}\right) \phi\left(g k_{\varphi}\right) & =\tau_{n}\left(k_{\varphi}\right) \phi\left(k_{\theta} a_{t} n_{x}\right) \\
& =\tau_{n}\left(k_{\varphi}\right) \xi_{n, s}\left(a_{t}\right)^{-1} \phi\left(k_{\theta}\right) \\
& =\tau_{n}\left(k_{\varphi}\right) \xi_{n, s}\left(a_{t}\right)^{-1} \psi\left(k_{\theta} \mu_{o}\right) \\
& =e^{-i n \varphi / 2} e^{-s t} \psi\left(\left(\begin{array}{cc}
1 & e^{i \theta} \\
0 & 1
\end{array}\right) k_{\theta}\right) \\
& =\left(\frac{-\bar{b} e^{i \theta / 2}+a e^{-i \theta / 2}}{\left|-\bar{b} e^{i \theta / 2}+a e^{-i \theta / 2}\right|}\right)^{n}\left|-\bar{b} e^{i \theta / 2}+a e^{-i \theta / 2}\right|^{s} e^{i n \theta / 2} \Phi\left(e^{i \theta}\right) \\
& =\frac{\left|\bar{a} e^{i \theta / 2}-b e^{-i \theta / 2}\right|^{n+s}}{\left(\bar{a} e^{i \theta / 2}-b e^{-i \theta / 2}\right)^{n}} e^{i n \theta / 2} \Phi\left(e^{i \theta}\right) \\
& =\frac{1}{\bar{a}^{n}}\left(\frac{e^{i \theta}}{e^{i \theta}-z}\right)^{n}\left(\frac{1-|z|^{2}}{\left|e^{i \theta}-z\right|^{2}}\right)^{-(n+s) / 2} \Phi\left(e^{i \theta}\right)
\end{aligned}
$$

On the other hand, we see that

$$
\begin{aligned}
f(g) & =f\left(\left(\begin{array}{cc}
a & b \\
\bar{b} & \bar{a}
\end{array}\right)\right) \\
& =f\left(\left(\begin{array}{cc}
1 & \frac{b}{\bar{a}} \\
0 & 1
\end{array}\right)\left(\begin{array}{cc}
\frac{1}{\bar{a}} & 0 \\
\bar{b} & \bar{a}
\end{array}\right)\right) \\
& =\frac{1}{\bar{a}^{n}} f\left(\left(\begin{array}{ll}
1 & \frac{b}{\bar{a}} \\
0 & 1
\end{array}\right)\right)=\frac{1}{\bar{a}^{n}} F(z) .
\end{aligned}
$$

Recall that in Section 2 we showed

$$
\frac{d \varphi}{d \theta}=\frac{1}{\left|\bar{a} e^{i \theta}-b\right|^{2}}=\frac{1-|z|^{2}}{\left|e^{i \theta}-z\right|^{2}}
$$

Therefore we finally obtain

$$
F(z)=\frac{1}{4 \pi} \int_{0}^{4 \pi}\left(\frac{e^{i \theta}}{e^{i \theta}-z}\right)^{n}\left(\frac{1-|z|^{2}}{\left|e^{i \theta}-z\right|^{2}}\right)^{-(n+s) / 2} \Phi\left(e^{i \theta}\right) \frac{d \varphi}{d \theta} d \theta
$$




$$
=\frac{1}{2 \pi} \int_{0}^{2 \pi}\left(\frac{e^{i \theta}}{e^{i \theta}-z}\right)^{n}\left(\frac{1-|z|^{2}}{\left|e^{i \theta}-z\right|^{2}}\right)^{(2-n-s) / 2} \Phi\left(e^{i \theta}\right) d \theta .
$$

In case $n=s=0$, we have the usual Poisson kernel function

$$
F(z)=\frac{1}{2 \pi} \int_{0}^{2 \pi} \frac{1-|z|^{2}}{\left|e^{i \theta}-z\right|^{2}} \Phi\left(e^{i \theta}\right) d \theta .
$$

In case $n=0$, replacing $s$ by $2-2 s$ we have the generalized Poisson kernel function

$$
F(z)=\frac{1}{2 \pi} \int_{0}^{2 \pi}\left(\frac{1-|z|^{2}}{\left|e^{i \theta}-z\right|^{2}}\right)^{s} \Phi\left(e^{i \theta}\right) d \theta
$$

which we mentioned in Section 2. In case $n=s=1$, putting $e^{i \theta}=\zeta$ we have the usual Cauchy kernel function

$$
F(z)=\frac{1}{2 \pi} \int_{0}^{2 \pi} \frac{e^{i \theta}}{e^{i \theta}-z} \Phi\left(e^{i \theta}\right) d \theta=\frac{1}{2 \pi i} \oint \frac{\Phi(\zeta)}{\zeta-z} d \zeta .
$$

\section{Computation of kernel functions}

We keep the notation of the previous section. Choose $\chi \in C^{\infty}(G)$ such that

$$
\begin{aligned}
& \chi(k \operatorname{man})=\chi(\operatorname{man}) \quad(k \in K, m \in M, a \in A, n \in N), \\
& \int_{M A N} \chi(\text { man }) d m d a d n=1
\end{aligned}
$$

For any $g \in G$, we put

$$
\begin{aligned}
& \kappa(g)=k m, \quad H(g)=\log a, \quad n(g)=n \\
& (g=k m a n, k \in K, m \in M, a \in A, n \in N) .
\end{aligned}
$$

It is easy to see that for any $k_{1}, k_{2} \in K$ and $m_{1}, m_{2} \in M$ with $k_{1} m_{1}=k_{2} m_{2}$ we have

$$
\tau\left(k_{1}\right) \xi\left(m_{1}\right)=\tau\left(k_{2}\right) \xi\left(m_{2}\right)
$$

Thus we can define

$$
\omega(k m)=\tau(k) \xi(m) \quad(k \in K, m \in M) .
$$


Then for any $g \in G$ we have

$$
\begin{aligned}
& f(g)=\int_{K} \tau(k) \phi(g k) d k \\
& =\int_{K M A N} \tau(k) \phi(g k) \chi(\operatorname{man}) d k d m d a d n \\
& =\int_{K M A N} \tau(k) \xi(m) \xi(a) \phi(g k m a n) \chi(k m a n) d k d m d a d n \\
& =\int_{K M A N} \omega(k m) \xi(a) e^{-2 \rho(\log a)} \phi(g k m a n) \chi(k m a n) e^{2 \rho(\log a)} d k d m d a d n \\
& =\int_{G} \omega\left(\kappa\left(g_{1}\right)\right) \xi\left(\exp H\left(g_{1}\right)\right) e^{-2 \rho\left(H\left(g_{1}\right)\right)} \phi\left(g g_{1}\right) \chi\left(g_{1}\right) d g_{1} \\
& =\int_{G} \omega\left(\kappa\left(g^{-1} g_{1}\right)\right) \xi\left(\exp H\left(g^{-1} g_{1}\right)\right) e^{-2 \rho\left(H\left(g^{-1} g_{1}\right)\right)} \phi\left(g_{1}\right) \chi\left(g^{-1} g_{1}\right) d g_{1} \\
& =\int_{K M A N} \omega\left(\kappa\left(g^{-1} k m a n\right)\right) \xi\left(\exp H\left(g^{-1} k m a n\right)\right) e^{-2 \rho\left(H\left(g^{-1} k m a n\right)\right)} \\
& \times \phi(k m a n) \chi\left(g^{-1} k m a n\right) e^{2 \rho(\log a)} d k d m d a d n \\
& =\int_{K M A N} \omega\left(\kappa\left(g^{-1} k\right) m\right) \xi\left(\exp \left(H\left(g^{-1} k\right)+\log a\right)\right) e^{-2 \rho\left(H\left(g^{-1} k\right)+\log a\right)} \xi(m a)^{-1} \\
& \times \phi(k) \chi\left(\kappa\left(g^{-1} k\right) \exp \left(H\left(g^{-1} k\right)\right) n\left(g^{-1} k\right) m a n\right) e^{2 \rho(\log a)} d k d m d a d n \\
& =\int_{K M A N} \omega\left(\kappa\left(g^{-1} k\right)\right) \xi\left(\exp H\left(g^{-1} k\right)\right) e^{-2 \rho\left(H\left(g^{-1} k\right)\right)} \\
& \times \phi(k) \chi\left(\kappa\left(g^{-1} k\right) m \exp \left(H\left(g^{-1} k\right)\right) a n\right) d k d m d a d n \\
& =\int_{K M A N} \omega\left(\kappa\left(g^{-1} k\right)\right) \xi\left(\exp H\left(g^{-1} k\right)\right) e^{-2 \rho\left(H\left(g^{-1} k\right)\right)} \phi(k) \chi(\operatorname{man}) d k d m d a d n \\
& =\int_{K} \omega\left(\kappa\left(g^{-1} k\right)\right) \xi\left(\exp H\left(g^{-1} k\right)\right) e^{-2 \rho\left(H\left(g^{-1} k\right)\right)} \phi(k) d k .
\end{aligned}
$$

On the other hand, for any $g \in G$ and $k \in K$ we get

$$
\begin{aligned}
g & =\exp g[0] u(g)=(\exp z) u(g), \\
k \mu_{o} & =k \mu_{o} k^{-1} k=\left(\exp k\left[u_{o}\right]\right) k=(\exp u) k
\end{aligned}
$$

This implies that

$$
\begin{aligned}
& f(g)=h((\exp z) u(g))=\tau(u(g))^{-1} h(\exp z)=\tau(u(g))^{-1} F(z) \\
& \phi(k)=\psi\left(k \gamma_{o}\right)=\eta(k)^{-1} \psi(\exp u)=\tau(k)^{-1} \Phi(u)
\end{aligned}
$$


It follows that

$$
\begin{aligned}
F(z) & =\int_{K} \tau(u(g)) \omega\left(\kappa\left(g^{-1} k\right)\right) \xi\left(\exp H\left(g^{-1} k\right)\right) e^{-2 \rho\left(H\left(g^{-1} k\right)\right)} \tau(k)^{-1} \Phi(u) d u \\
& =\int_{K} \tau\left(u\left(g k^{-1}\right)\right) \omega\left(\kappa\left(g^{-1} k\right)\right) \xi\left(\exp H\left(g^{-1} k\right)\right) e^{-2 \rho\left(H\left(g^{-1} k\right)\right)} \Phi(u) d u
\end{aligned}
$$

TheOREM 1. The generalized Poisson-Cauchy transform is an integral operator with the kernel function:

$$
\begin{aligned}
\mathcal{K}_{\tau, \eta}(z, u)= & \tau\left(u\left(g k^{-1}\right)\right) \omega\left(\kappa\left(g^{-1} k\right)\right) \xi\left(\exp H\left(g^{-1} k\right)\right) e^{-2 \rho\left(H\left(g^{-1} k\right)\right)} \\
& \left(z=g[0], u=k\left[u_{o}\right]\right)
\end{aligned}
$$

which we call the generalized Poisson-Cauchy kernel function.

\subsection{Type I}

Fix any $\ell \in \boldsymbol{Z}$ and $s \in \boldsymbol{C}$ we define characters $\tau_{\ell}, \eta_{\ell, s}$ of $U$ and a character $\xi_{\ell, s}$ of $P$ by

$$
\begin{aligned}
\tau_{\ell}: U \ni\left(\begin{array}{cc}
\alpha & 0 \\
\zeta & \delta
\end{array}\right) \longmapsto(\operatorname{det}(\delta))^{\ell} \in C^{*} \\
\eta_{\ell, s}: U \ni\left(\begin{array}{cc}
\alpha & 0 \\
\zeta & \delta
\end{array}\right) \longmapsto\left(\frac{\operatorname{det}(\delta)}{|\operatorname{det}(\delta)|}\right)^{\ell}|\operatorname{det}(\delta)|^{s} \in C^{*} \\
\xi_{\ell, s}: P \ni p \longmapsto \eta_{\ell, s}\left(\mu_{o}^{-1} p \mu_{o}\right) \in C^{*}
\end{aligned}
$$

For any $g \in G$ and $k \in K$ denote

$$
\begin{aligned}
& g=\left(\begin{array}{ll}
a & b \\
c & d
\end{array}\right), \\
& k=\left(\begin{array}{cc}
k_{1} & 0 \\
0 & k_{2}
\end{array}\right) .
\end{aligned}
$$

Then since $g^{*} I_{n, m} g=I_{n, m}$ we have

$$
\begin{aligned}
g k^{-1} & =\left(\begin{array}{cc}
a k_{1}^{-1} & b k_{2}^{-1} \\
c k_{1}^{-1} & d k_{2}^{-1}
\end{array}\right) \\
g^{-1} k & =\left(\begin{array}{cc}
a^{*} k_{1} & -c^{*} k_{2} \\
-b^{*} k_{1} & d^{*} k_{2}
\end{array}\right) .
\end{aligned}
$$

It is easy to see that

$$
\tau_{\ell}\left(u\left(g k^{-1}\right)\right)=\left(\operatorname{det}\left(d k_{2}^{-1}\right)\right)^{\ell} .
$$


Put

$$
g^{-1} k=\widehat{k} m a n
$$

where

$$
\begin{aligned}
\widehat{k} & =\left(\begin{array}{cc}
\widehat{k}_{1} & 0 \\
0 & \widehat{k}_{2}
\end{array}\right), \\
m & =\left(\begin{array}{ccc}
m_{1} & 0 & m_{2} \\
0 & m_{3} & 0 \\
m_{2} & 0 & m_{1}
\end{array}\right), \\
a & =\left(\begin{array}{ccc}
\operatorname{ch} t I_{m} & 0 & \operatorname{sh} t I_{m} \\
0 & I_{n-m} & 0 \\
\operatorname{sh} t I_{m} & 0 & \operatorname{ch} t I_{m}
\end{array}\right), \\
n & =\left(\begin{array}{ccc}
I_{m}+\xi-\frac{1}{2} \eta \eta^{*} & \eta \\
-\eta^{*} & I_{n-m} & -\xi+\frac{1}{2} \eta \eta^{*} \\
\xi-\frac{1}{2} \eta \eta^{*} & \eta & I_{m}-\xi+\frac{1}{2} \eta \eta^{*}
\end{array}\right)
\end{aligned}
$$

Put

$$
p_{o}=\left(\begin{array}{c}
I_{m} \\
0 \\
I_{m}
\end{array}\right)
$$

Then the last $(m, m)$-components of $\widehat{k} m a n p_{o}$ is equal to $\widehat{k}_{2}\left(m_{1}+m_{2}\right) e^{t}$. Comparing the last $(m, m)$-components of $\widehat{k} m a n p_{o}$ with that of $g^{-1} k p_{o}$, we have

$$
\widehat{k}_{2}\left(m_{1}+m_{2}\right) e^{t}=\left(-b^{*} u+d^{*}\right) k_{2}, \quad \text { where } u=k_{1}\left(\begin{array}{c}
I_{m} \\
0
\end{array}\right) k_{2}^{-1} \text {. }
$$

It follows that

$$
\begin{aligned}
e^{m t} & =\left|\operatorname{det}\left(-b^{*} u+d^{*}\right)\right|=\left|\operatorname{det}\left(d-u^{*} b\right)\right| \\
e^{-2 \rho\left(H\left(g^{-1} k\right)\right)} & =e^{-2 m n t}=\left|\operatorname{det}\left(d-u^{*} b\right)\right|^{-2 n} \\
\omega_{\ell, s}\left(\kappa\left(g^{-1} k\right)\right) & =\left(\frac{\operatorname{det}\left(\left(-b^{*} u+d^{*}\right) k_{2}\right)}{\left|\operatorname{det}\left(\left(-b^{*} u+d^{*}\right) k_{2}\right)\right|}\right)^{\ell}=\left(\frac{\left|\operatorname{det}\left(d-u^{*} b\right)\right| k_{2}}{\operatorname{det}\left(d-u^{*} b\right)}\right)^{\ell}, \\
\xi_{\ell, s}\left(\exp \left(H\left(g^{-1} k\right)\right)\right) & =\left|\operatorname{det}\left(\left(-b^{*} u+d^{*}\right) k_{2}\right)\right|^{s}=\left|\operatorname{det}\left(d-u^{*} b\right)\right|^{s} .
\end{aligned}
$$


Comparing the last $(m, m)$-components of both sides of $g^{*} I_{n, m} g=I_{n, m}$, we have $d^{*} d-b^{*} b=I_{m}$. Since $\frac{b}{d}=z$ we get

$$
\operatorname{det}\left(d^{*} d\right)=\frac{1}{\operatorname{det}\left(I_{m}-z^{*} z\right)}
$$

Thus we finally obtain

$$
\begin{aligned}
\mathcal{K}_{\tau_{\ell}, \eta_{\ell, s}}(z, u) & =\tau_{\ell}\left(u\left(g k^{-1}\right)\right) \omega_{\ell, s}\left(\kappa\left(g^{-1} k\right)\right) \xi_{\ell, s}\left(\exp H\left(g^{-1} k\right)\right) e^{-2 \rho\left(H\left(g^{-1} k\right)\right)} \\
& =\frac{1}{\left(\operatorname{det}\left(I_{m}-u^{*} z\right)\right)^{\ell}}\left(\frac{\operatorname{det}\left(I_{m}-z^{*} z\right)}{\left|\operatorname{det}\left(I_{m}-u^{*} z\right)\right|^{2}}\right)^{n-(\ell+s) / 2}
\end{aligned}
$$

Theorem 2. The generalized Poisson-Cauchy kernel function for Type I is given by

$$
\mathcal{K}_{\tau_{\ell}, \eta_{\ell, s}}(z, u)=\frac{1}{\left(\operatorname{det}\left(I_{m}-u^{*} z\right)\right)^{\ell}}\left(\frac{\operatorname{det}\left(I_{m}-z^{*} z\right)}{\left|\operatorname{det}\left(I_{m}-u^{*} z\right)\right|^{2}}\right)^{n-(\ell+s) / 2}
$$

In case $\ell=s=0$ our kernel function coincides with the Poisson kernel function. (See [3], page 98)

In case $\ell=s=n$ our kernel function coincides with the Cauchy kernel function. (See [3], page 94)

\subsection{Type II}

Fix any $\ell \in Z$ and $s \in C$ we define characters $\tau_{\ell}, \eta_{\ell, s}$ of $U$ and a character $\xi_{\ell, s}$ of $P$ by

$$
\begin{aligned}
\tau_{\ell} & : U \ni\left(\begin{array}{cc}
{ }^{t} \delta^{-1} & 0 \\
\zeta & \delta
\end{array}\right) \longmapsto(\operatorname{det}(\delta))^{\ell} \in C^{*} \\
\eta_{\ell, s} & : U \ni\left(\begin{array}{cc}
{ }^{t} \delta^{-1} & 0 \\
\zeta & \delta
\end{array}\right) \longmapsto\left(\frac{\operatorname{det}(\delta)}{|\operatorname{det}(\delta)|}\right)^{\ell}|\operatorname{det}(\delta)|^{s} \in C^{*} \\
\xi_{\ell, s} & : P \ni p \longmapsto \eta_{\ell, s}\left(\mu_{o}^{-1} p \mu_{o}\right) \in C^{*}
\end{aligned}
$$

For any $g \in G$ and $k \in K$, we put

$$
\begin{aligned}
& g=\left(\begin{array}{cc}
a & b \\
\bar{b} & \bar{a}
\end{array}\right), \\
& k=\left(\begin{array}{cc}
k_{1} & 0 \\
0 & k_{1}
\end{array}\right) .
\end{aligned}
$$


Then since $g^{*} I_{n, n} g=I_{n, n}$, we have

$$
\begin{aligned}
& g k^{-1}=\left(\begin{array}{ll}
a k_{1}^{-1} & b \overline{k_{1}} \\
\bar{b} k_{1}^{-1} & \bar{a} \bar{k}_{1}^{-1}
\end{array}\right), \\
& g^{-1} k=\left(\begin{array}{cc}
a^{*} k_{1} & -{ }^{t} b \overline{k_{1}} \\
-b^{*} k_{1} & { }^{t} a \overline{k_{1}}
\end{array}\right) .
\end{aligned}
$$

It is easy to see that

$$
\tau_{\ell}\left(u\left(g k^{-1}\right)\right)=\left(\operatorname{det}\left(\bar{a}{\overline{k_{1}}}^{-1}\right)\right)^{\ell}
$$

Put

$$
g^{-1} k=\widehat{k} m a n
$$

where

$$
\begin{aligned}
\widehat{k} & =\left(\begin{array}{cc}
\widehat{k}_{1} & 0 \\
0 & \widehat{k}_{2}
\end{array}\right), \\
m & =\left(\begin{array}{cc}
m_{1} & m_{2} \\
m_{2} & m_{1}
\end{array}\right), \\
a & =\left(\begin{array}{cc}
\operatorname{ch} t I_{n} & \operatorname{sh} t I_{n} \\
\operatorname{sh} t I_{n} & \operatorname{ch} t I_{n}
\end{array}\right), \\
n & =\left(\begin{array}{cc}
I_{n}+i \xi & -i \xi \\
i \xi & I_{n}-i \xi
\end{array}\right) .
\end{aligned}
$$

Put

$$
p_{o}=\left(\begin{array}{c}
I_{n} \\
I_{n}
\end{array}\right)
$$

Then the last $(n, n)$-components of $\widehat{k} m a n p_{o}$ is equal to $e^{t} \widehat{\widehat{k}}_{1}\left(m_{1}+m_{2}\right)$. Comparing the last $(n, n)$-components of $\widehat{k} m a n p_{o}$ with that of $g^{-1} k p_{o}$, we have

$$
e^{t} \overline{\widehat{k}_{1}}\left(m_{1}+m_{2}\right)=\left(-b^{*} u+{ }^{t} a\right) \overline{k_{1}}, \quad \text { where } u=k_{1}{\overline{k_{1}}}^{-1} \text {. }
$$

It follows that

$$
\begin{aligned}
e^{n t} & =\left|\operatorname{det}\left(-b^{*} u+{ }^{t} a\right)\right|=\left|\operatorname{det}\left(\bar{a}-u^{*} b\right)\right|, \\
e^{-2 \rho\left(H\left(g^{-1} k\right)\right)} & =e^{-n(n+1) t}=\left|\operatorname{det}\left(\bar{a}-u^{*} b\right)\right|^{-(n+1)}, \\
\omega_{\ell, s}\left(\kappa\left(g^{-1} k\right)\right) & =\left(\frac{\operatorname{det}\left(\left(-b^{*} u+{ }^{t} a\right) k_{2}\right)}{\left|\operatorname{det}\left(\left(-b^{*} u+{ }^{t} a\right) \overline{k_{1}}\right)\right|}\right)^{\ell}=\left(\frac{\left|\operatorname{det}\left(\bar{a}-u^{*} b\right)\right| \overline{k_{1}}}{\operatorname{det}\left(\bar{a}-u^{*} b\right)}\right)^{\ell}, \\
\xi_{\ell, s}\left(\exp \left(H\left(g^{-1} k\right)\right)\right) & =\left|\operatorname{det}\left(\left(-b^{*} u+{ }^{t} a\right) \overline{k_{1}}\right)\right|^{s}=\left|\operatorname{det}\left(\bar{a}-u^{*} b\right)\right|^{s} .
\end{aligned}
$$


Comparing the last $(n, n)$-components of both sides of $g^{*} I_{n, n} g=I_{n, n}$ we have ${ }^{t} a \bar{a}-b^{*} b=I_{n}$. Since $\frac{b}{\bar{a}}=z$ we get

$$
\operatorname{det}\left({ }^{t} a \bar{a}\right)=\frac{1}{\operatorname{det}\left(I_{n}-z^{*} z\right)}
$$

Thus we finally obtain

$$
\begin{aligned}
\mathcal{K}_{\tau_{\ell}, \eta_{\ell, s}}(z, u) & =\tau_{\ell}\left(u\left(g k^{-1}\right)\right) \omega_{\ell, s}\left(\kappa\left(g^{-1} k\right)\right) \xi_{\ell, s}\left(\exp H\left(g^{-1} k\right)\right) e^{-2 \rho\left(H\left(g^{-1} k\right)\right)} \\
& =\frac{1}{\left(\operatorname{det}\left(I_{n}-u^{*} z\right)\right)^{\ell}}\left(\frac{\operatorname{det}\left(I_{n}-z^{*} z\right)}{\left|\operatorname{det}\left(I_{n}-u^{*} z\right)\right|^{2}}\right)^{(n+1-\ell-s) / 2} \\
& =\frac{1}{\left(\operatorname{det}\left(I_{n}-\bar{u} z\right)\right)^{\ell}}\left(\frac{\operatorname{det}\left(I_{n}-\bar{z} z\right)}{\left|\operatorname{det}\left(I_{n}-\bar{u} z\right)\right|^{2}}\right)^{(n+1-\ell-s) / 2}
\end{aligned}
$$

Theorem 3. The generalized Poisson-Cauchy kernel function for Type II is given by

$$
\mathcal{K}_{\tau_{\ell}, \eta_{\ell, s}}(z, u)=\frac{1}{\left(\operatorname{det}\left(I_{n}-\bar{u} z\right)\right)^{\ell}}\left(\frac{\operatorname{det}\left(I_{n}-\bar{z} z\right)}{\left|\operatorname{det}\left(I_{n}-\bar{u} z\right)\right|^{2}}\right)^{(n+1-\ell-s) / 2} .
$$

In case $\ell=s=0$ our kernel function coincides with the Poisson kernel function. (See [3], page 99)

In case $\ell=s=\frac{n+1}{2}$ our kernel function coincides with the Cauchy kernel function. (See [3], page 95)

\subsection{Type III}

Fix any $\ell \in \boldsymbol{Z}$ and $s \in \boldsymbol{C}$ we define characters $\tau_{\ell}, \eta_{\ell, s}$ of $U$ and a character $\xi_{\ell, s}$ of $P$ by

$$
\begin{aligned}
\tau_{\ell} & : U \ni\left(\begin{array}{cc}
{ }^{t} \delta^{-1} & 0 \\
\zeta & \delta
\end{array}\right) \longmapsto(\operatorname{det}(\delta))^{\ell} \in C^{*} \\
\eta_{\ell, s} & : U \ni\left(\begin{array}{cc}
{ }^{t} \delta^{-1} & 0 \\
\zeta & \delta
\end{array}\right) \longmapsto\left(\frac{\operatorname{det}(\delta)}{|\operatorname{det}(\delta)|}\right)^{\ell}|\operatorname{det}(\delta)|^{s} \in C^{*}, \\
\xi_{\ell, s} & : P \ni p \longmapsto \eta_{\ell, s}\left(\mu_{o}^{-1} p \mu_{o}\right) \in C^{*}
\end{aligned}
$$

For any $g \in G$ and $k \in K$ denote

$$
\begin{aligned}
& g=\left(\begin{array}{cc}
a & b \\
-\bar{b} & \bar{a}
\end{array}\right), \\
& k=\left(\begin{array}{cc}
k_{1} & 0 \\
0 & k_{1}
\end{array}\right) .
\end{aligned}
$$


Then since $g^{*} I_{n, n} g=I_{n, n}$ we have

$$
\begin{aligned}
g k^{-1} & =\left(\begin{array}{cc}
a k_{1}^{-1} & b{\overline{k_{1}}}^{-1} \\
-\bar{b} k_{1}^{-1} & \bar{a}{\overline{k_{1}}}^{-1}
\end{array}\right) \\
g^{-1} k & =\left(\begin{array}{cc}
a^{*} k_{1} & { }^{t} b \overline{k_{1}} \\
-b^{*} k_{1} & { }^{t} a \overline{k_{1}}
\end{array}\right) .
\end{aligned}
$$

It is easy to see that

$$
\tau_{\ell}\left(u\left(g k^{-1}\right)\right)=\left(\operatorname{det}\left(\bar{a}{\overline{k_{1}}}^{-1}\right)\right)^{\ell}
$$

\subsubsection{Case n: even}

Put $m=\frac{n}{2}$. Put

$$
g^{-1} k=\widehat{k} m a n
$$

where

$$
\begin{aligned}
\widehat{k} & =\left(\begin{array}{cc}
\widehat{k}_{1} & 0 \\
0 & \overline{\hat{k}_{1}}
\end{array}\right), \\
\sigma & =\left(\begin{array}{cc}
0 & I_{m} \\
-I_{m} & 0
\end{array}\right), \\
m & =\left(\begin{array}{cc}
m_{1} & m_{2} \\
-\overline{m_{2}} & \overline{m_{1}}
\end{array}\right), \\
a & =\left(\begin{array}{cc}
\operatorname{ch} t I_{n} & \operatorname{sh} t \sigma \\
-\operatorname{sh} t \sigma & \operatorname{ch} t I_{n}
\end{array}\right), \\
n & =\left(\begin{array}{cc}
I_{n}+\xi & -\xi \sigma \\
\bar{\xi} \sigma & I_{n}+\bar{\xi}
\end{array}\right) .
\end{aligned}
$$

Put

$$
p_{o}=\left(\begin{array}{c}
\sigma \\
I_{n}
\end{array}\right)
$$

Then the last $(n, n)$-components of $\widehat{k} m a n p_{o}$ is equal to $e^{t} \overline{\widehat{k}_{1}}\left(\overline{m_{1}}-\overline{m_{2}} \sigma\right)$. Comparing the last $(n, n)$-components of $\widehat{k} m a n p_{o}$ with that of $g^{-1} k p_{o}$, we have

$$
e^{t} \overline{\widehat{k}_{1}}\left(\overline{m_{1}}-\overline{m_{2}} \sigma\right)=\left(-b^{*} u+{ }^{t} a\right) \overline{k_{1}}, \quad \text { where } u=k_{1} \sigma{\overline{k_{1}}}^{-1}
$$


It follows that

$$
\begin{aligned}
e^{n t} & =\left|\operatorname{det}\left(-b^{*} u+{ }^{t} a\right)\right|=\left|\operatorname{det}\left(\bar{a}-u^{*} b\right)\right|, \\
e^{-2 \rho\left(H\left(g^{-1} k\right)\right)} & =e^{-n(n-1) t}=\left|\operatorname{det}\left(\bar{a}-u^{*} b\right)\right|^{-(n-1)}, \\
\omega_{\ell, s}\left(\kappa\left(g^{-1} k\right)\right) & =\left(\frac{\operatorname{det}\left(\left(-b^{*} u+{ }^{t} a\right) \overline{k_{1}}\right)}{\left|\operatorname{det}\left(\left(-b^{*} u+{ }^{t} a\right) \overline{k_{1}}\right)\right|}\right)^{\ell}=\left(\frac{\left|\operatorname{det}\left(\bar{a}-u^{*} b\right)\right| \overline{k_{1}}}{\operatorname{det}\left(\bar{a}-u^{*} b\right)}\right)^{\ell}, \\
\xi_{\ell, s}\left(\exp \left(H\left(g^{-1} k\right)\right)\right) & =\left|\operatorname{det}\left(\left(-b^{*} u+{ }^{t} a\right) \overline{k_{1}}\right)\right|^{s}=\left|\operatorname{det}\left(\bar{a}-u^{*} b\right)\right|^{s} .
\end{aligned}
$$

Comparing the last $(n, n)$-components of both sides of $g^{*} I_{n, n} g=I_{n, n}$, we have ${ }^{t} a \bar{a}-b^{*} b=I_{n}$. Since $\frac{b}{\bar{a}}=z$ we get

$$
\operatorname{det}\left({ }^{t} a \bar{a}\right)=\frac{1}{\operatorname{det}\left(I_{n}-z^{*} z\right)}
$$

Thus we finally obtain

$$
\begin{aligned}
\mathcal{K}_{\tau_{\ell}, \eta_{\ell, s}}(z, u) & =\tau_{\ell}\left(u\left(g k^{-1}\right)\right) \omega_{\ell, s}\left(\kappa\left(g^{-1} k\right)\right) \xi_{\ell, s}\left(\exp H\left(g^{-1} k\right)\right) e^{-2 \rho\left(H\left(g^{-1} k\right)\right)} \\
& =\frac{1}{\left(\operatorname{det}\left(I_{n}-u^{*} z\right)\right)^{\ell}}\left(\frac{\operatorname{det}\left(I_{n}-z^{*} z\right)}{\left|\operatorname{det}\left(I_{n}-u^{*} z\right)\right|^{2}}\right)^{(n-1-\ell-s) / 2} \\
& =\frac{1}{\left(\operatorname{det}\left(I_{n}+\bar{u} z\right)\right)^{\ell}}\left(\frac{\operatorname{det}\left(I_{n}+\bar{z} z\right)}{\left|\operatorname{det}\left(I_{n}+\bar{u} z\right)\right|^{2}}\right)^{(n-1-\ell-s) / 2}
\end{aligned}
$$

Theorem 4. The generalized Poisson-Cauchy kernel function for Type $\mathrm{II}$ for even $n$ is given by

$$
\mathcal{K}_{\tau_{\ell}, \eta_{\ell, s}}(z, u)=\frac{1}{\left(\operatorname{det}\left(I_{n}+\bar{u} z\right)\right)^{\ell}}\left(\frac{\operatorname{det}\left(I_{n}+\bar{z} z\right)}{\left|\operatorname{det}\left(I_{n}+\bar{u} z\right)\right|^{2}}\right)^{(n-1-\ell-s) / 2} .
$$

In case $\ell=s=0$ our kernel function coincides with the Poisson kernel function. (See [3], page 99)

In case $\ell=s=(n-1) / 2$ our kernel function coincides with the Cauchy kernel function. (See [3], page 95)

\subsubsection{Case n: odd}

Put $m=\frac{n-1}{2}$. Put

$$
g^{-1} k=\widehat{k} \operatorname{man}
$$


where

$$
\begin{aligned}
\widehat{k} & =\left(\begin{array}{cc}
\widehat{k}_{1} & 0 \\
0 & \overline{\widehat{k}_{1}}
\end{array}\right) \\
\sigma & =\left(\begin{array}{ccc}
0 & 0 & I_{m} \\
0 & 0 & 0 \\
-I_{m} & 0 & 0
\end{array}\right), \\
m & =\left(\begin{array}{cc}
m_{1} & m_{2} \\
-\overline{m_{2}} & \overline{m_{1}}
\end{array}\right) \\
a & =\left(\begin{array}{cc}
a(t) & b(t) \\
-b(t) & a(t)
\end{array}\right), \quad a(t)=\left(\begin{array}{ccc}
\operatorname{ch} t I_{m} & 0 & 0 \\
0 & 1 & 0 \\
0 & 0 & \operatorname{ch} t I_{m}
\end{array}\right), \quad b(t)=\operatorname{sh} t \sigma \\
n & =\left(\begin{array}{cc}
I_{n}+\xi & -\xi \sigma \\
\bar{\xi} \sigma & I_{n}+\bar{\xi}
\end{array}\right) .
\end{aligned}
$$

Put

$$
p_{o}=\left(\begin{array}{c}
\sigma \\
I_{n}
\end{array}\right)
$$

Then the last $(n, n)$-components of $\widehat{k} m a n p_{o}$ is equal to $\overline{\widehat{k}_{1}}\left(\overline{m_{1}}-\overline{m_{2}} \sigma\right)$ $\left(\begin{array}{ccc}e^{t} I_{m} & 0 & 0 \\ 0 & 1 & 0 \\ 0 & 0 & e^{t} I_{m}\end{array}\right)$. Comparing the last $(n, n)$-components of $\widehat{k} m a n p_{o}$ with that of $g^{-1} k p_{o}$, we have

$$
\overline{\widehat{k}_{1}}\left(\overline{m_{1}}-\overline{m_{2}} \sigma\right)\left(\begin{array}{ccc}
e^{t} I_{m} & 0 & 0 \\
0 & 1 & 0 \\
0 & 0 & e^{t} I_{m}
\end{array}\right)=\left(-b^{*} u+{ }^{t} a\right) \overline{k_{1}} \text {, where } u=k_{1} \sigma{\overline{k_{1}}}^{-1} .
$$

It follows that

$$
\begin{aligned}
e^{(n-1) t} & =\left|\operatorname{det}\left(-b^{*} u+{ }^{t} a\right)\right|=\left|\operatorname{det}\left(\bar{a}-u^{*} b\right)\right|, \\
e^{-2 \rho\left(H\left(g^{-1} k\right)\right)} & =e^{-n(n-1) t}=\left|\operatorname{det}\left(\bar{a}-u^{*} b\right)\right|^{-n}, \\
\omega_{\ell, s}\left(\kappa\left(g^{-1} k\right)\right) & =\left(\frac{\operatorname{det}\left(\left(-b^{*} u+{ }^{t} a\right) \overline{k_{1}}\right)}{\left|\operatorname{det}\left(\left(-b^{*} u+{ }^{t} a\right) \overline{k_{1}}\right)\right|}\right)^{\ell}=\left(\frac{\left|\operatorname{det}\left(\bar{a}-u^{*} b\right)\right| \overline{k_{1}}}{\operatorname{det}\left(\bar{a}-u^{*} b\right)}\right)^{\ell}, \\
\xi_{\ell, s}\left(\exp \left(H\left(g^{-1} k\right)\right)\right) & =\left|\operatorname{det}\left(\left(-b^{*} u+{ }^{t} a\right) \overline{k_{1}}\right)\right|^{s}=\left|\operatorname{det}\left(\bar{a}-u^{*} b\right)\right|^{s} .
\end{aligned}
$$


Comparing the last $(n, n)$-components of both sides of $g^{*} I_{n, n} g=I_{n, n}$, we have ${ }^{t} a \bar{a}-b^{*} b=I_{n}$. Since $\frac{b}{\bar{a}}=z$ we get

$$
\operatorname{det}\left({ }^{t} a \bar{a}\right)=\frac{1}{\operatorname{det}\left(I_{n}-z^{*} z\right)}
$$

Thus we finally obtain

$$
\begin{aligned}
\mathcal{K}_{\tau_{\ell}, \eta_{\ell, s}}(z, u) & =\tau_{\ell}\left(u\left(g k^{-1}\right)\right) \omega_{\ell, s}\left(\kappa\left(g^{-1} k\right)\right) \xi_{\ell, s}\left(\exp H\left(g^{-1} k\right)\right) e^{-2 \rho\left(H\left(g^{-1} k\right)\right)} \\
& =\frac{1}{\left(\operatorname{det}\left(I_{n}-u^{*} z\right)\right)^{\ell}}\left(\frac{\operatorname{det}\left(I_{n}-z^{*} z\right)}{\left|\operatorname{det}\left(I_{n}-u^{*} z\right)\right|^{2}}\right)^{(n-\ell-s) / 2} \\
& =\frac{1}{\left(\operatorname{det}\left(I_{n}+\bar{u} z\right)\right)^{\ell}}\left(\frac{\operatorname{det}\left(I_{n}+\bar{z} z\right)}{\left|\operatorname{det}\left(I_{n}+\bar{u} z\right)\right|^{2}}\right)^{(n-\ell-s) / 2}
\end{aligned}
$$

TheOREM 5. The generalized Poisson-Cauchy kernel function for Type III for odd $n$ is given by

$$
\mathcal{K}_{\tau_{\ell}, \eta_{\ell, s}}(z, u)=\frac{1}{\left(\operatorname{det}\left(I_{n}+\bar{u} z\right)\right)^{\ell}}\left(\frac{\operatorname{det}\left(I_{n}+\bar{z} z\right)}{\left|\operatorname{det}\left(I_{n}+\bar{u} z\right)\right|^{2}}\right)^{(n-\ell-s) / 2}
$$

In case $\ell=s=0$ our kernel function coincides with the Poisson kernel function. (See [3], page 99)

In case $\ell=s=\frac{n}{2}$ our kernel function coincides with the Cauchy kernel function. (See [3], page 95)

\subsection{Type IV}

Fix any $\ell \in \boldsymbol{Z}$ and $s \in C$ we define characters $\tau_{\ell}, \eta_{\ell, s}$ of $U$ and a character $\xi_{\ell, s}$ of $P$ by

$$
\begin{gathered}
\tau_{\ell}: U=K_{c} P_{-} \ni \gamma^{-1}\left(\begin{array}{ccc}
\alpha & 0 & 0 \\
0 & \delta_{1} & 0 \\
0 & 0 & \delta_{2}
\end{array}\right) p_{-} \gamma \longmapsto\left(\delta_{2}\right)^{\ell} \in C^{*}, \\
\eta_{\ell, s}: U=K_{c} P_{-} \ni \gamma^{-1}\left(\begin{array}{ccc}
\alpha & 0 & 0 \\
0 & \delta_{1} & 0 \\
0 & 0 & \delta_{2}
\end{array}\right) p_{-} \gamma \longmapsto\left(\frac{\delta_{2}}{\left|\delta_{2}\right|}\right)^{\ell}\left|\delta_{2}\right|^{s} \in C^{*}, \\
\xi_{\ell, s}: P \ni p \longmapsto \eta_{\ell, s}\left(\mu_{o}^{-1} p \mu_{o}\right) \in C^{*}
\end{gathered}
$$


For any $g \in G$ and $k \in K$ denote

$$
\begin{aligned}
g & =\left(\begin{array}{ll}
a & b \\
c & d
\end{array}\right), \\
k & =\left(\begin{array}{cc}
k_{1} & 0 \\
0 & k_{2}
\end{array}\right), \text { where } k_{2}=\left(\begin{array}{cc}
\cos \theta & \sin \theta \\
-\sin \theta & \cos \theta
\end{array}\right) .
\end{aligned}
$$

Then since $g^{*} I_{n, 2} g=I_{n, 2}$ we have

$$
\begin{aligned}
g k^{-1} & =\left(\begin{array}{cc}
a k_{1}^{-1} & b k_{2}^{-1} \\
c k_{1}^{-1} & d k_{2}^{-1}
\end{array}\right), \\
g^{-1} k & =\left(\begin{array}{cc}
{ }^{t} a k_{1} & -{ }^{t} c k_{2} \\
-{ }^{t} b k_{1} & { }^{t} d k_{2}
\end{array}\right) .
\end{aligned}
$$

It is easy to see that

$$
\tau_{\ell}\left(u\left(g k^{-1}\right)\right)=\left(\frac{1}{2}(-i, 1) d k_{2}^{-1}\left(\begin{array}{c}
i \\
1
\end{array}\right)\right)^{\ell}
$$

Put

$$
g^{-1} k=\widehat{k} m a n
$$

where

$$
\begin{aligned}
& k=\left(\begin{array}{cc}
\widehat{k}_{1} & 0 \\
0 & \widehat{k}_{2}
\end{array}\right), \text { where } \widehat{k}_{2}=\left(\begin{array}{cc}
\cos \varphi & \sin \varphi \\
-\sin \varphi & \cos \varphi
\end{array}\right) \\
& m=\left(\begin{array}{ccc}
m_{1} & 0 & 0 \\
0 & m_{2} & 0 \\
0 & 0 & m_{1}
\end{array}\right), \\
& a=\left(\begin{array}{ccc}
\operatorname{ch} t & 0 & \operatorname{sh} t \\
0 & I_{n} & 0 \\
\operatorname{sh} t & 0 & \operatorname{ch} t
\end{array}\right), \\
& n=\left(\begin{array}{ccc}
1+\xi-\frac{1}{2} \eta^{t} \eta & \eta & -\xi+\frac{1}{2} \eta^{t} \eta \\
-{ }^{t} \eta & I_{n} & { }^{t} \eta \\
\xi-\frac{1}{2} \eta^{t} \eta & \eta & 1-\xi+\frac{1}{2} \eta^{t} \eta
\end{array}\right)
\end{aligned}
$$


Put

$$
p_{o}=\left(\begin{array}{l}
x_{o} \\
y_{o}
\end{array}\right), \quad x_{o}=\left(\begin{array}{l}
1 \\
0
\end{array}\right) \in C^{n}, \quad y_{o}=\left(\begin{array}{l}
0 \\
1
\end{array}\right) \in C^{2}
$$

Then $(0, \ldots, 0,-i, 1) \widehat{k} m a n p_{o}$ is equal to $m_{1} e^{t} e^{-i \varphi}$. Comparing $(0, \ldots, 0,-i, 1) \widehat{k} m a n p_{o}$ with $(0, \ldots, 0,-i, 1) g^{-1} k p_{o}$, we have

$$
\begin{aligned}
m_{1} e^{t} e^{-i \varphi} & =(-i, 1)\left(-{ }^{t} b k_{1} x_{o}+{ }^{t} d k_{2} y_{o}\right) \\
& =(-i, 1)\left(-{ }^{t} b x+{ }^{t} d\left(\begin{array}{c}
\sin \theta \\
\cos \theta
\end{array}\right)\right) .
\end{aligned}
$$

We put $\Delta=\frac{1}{2}(-i, 1) d\left(\begin{array}{c}i \\ 1\end{array}\right)$. Then we obtain

$$
\begin{aligned}
m_{1} e^{t} e^{i \varphi} & =\left((\sin \theta, \cos \theta) d-{ }^{t} x b\right)\left(\begin{array}{c}
i \\
1
\end{array}\right) \\
& =\left(\frac{1}{2}\left(-i\left(e^{i \theta}-e^{-i \theta}\right), e^{i \theta}+e^{-i \theta}\right) d-{ }^{t} x b\right)\left(\begin{array}{c}
i \\
1
\end{array}\right) \\
& =\left(\frac{1}{2}\left((-i, 1) e^{i \theta}+(i, 1) e^{-i \theta}\right) d-{ }^{t} x b\right)\left(\begin{array}{c}
i \\
1
\end{array}\right) \\
& =\frac{1}{2}\left(e^{i \theta}(-i, 1) d\left(\begin{array}{c}
i \\
1
\end{array}\right)+e^{-i \theta}(i, 1) d\left(\begin{array}{c}
i \\
1
\end{array}\right)-2{ }^{t} x b\left(\begin{array}{c}
i \\
1
\end{array}\right)\right) \\
& =\left(e^{i \theta}+\frac{1}{2} e^{-i \theta}(i, 1) d\left(\begin{array}{c}
i \\
1
\end{array}\right) \Delta^{-1}-{ }^{t} x b\left(\begin{array}{c}
i \\
1
\end{array}\right) \Delta^{-1}\right) \Delta \\
& =\left(e^{i \theta}+e^{-i \theta t} z z-2{ }^{t} x z\right) \Delta \\
& =e^{-i \theta}\left(e^{2 i \theta}+{ }^{t} z z-2 e^{i \theta t} x z\right) \Delta \\
& =e^{-i \theta}\left({ }^{t} u u+{ }^{t} z z-2{ }^{t} u u z\right) \Delta \\
& =e^{-i \theta}\left({ }^{t}(u-z)(u-z)\right) \Delta
\end{aligned}
$$

Since ${ }^{t} g I_{n, 2} g=I_{n, 2}$ we get ${ }^{t} d d-{ }^{t} b b=I_{2}$ which implies

$$
\begin{aligned}
2 & =(-i, 1)^{t} d d\left(\begin{array}{c}
i \\
1
\end{array}\right)-(-i, 1)^{t} b b\left(\begin{array}{c}
i \\
1
\end{array}\right) \\
& =(-i, 1)^{t} d \frac{1}{2}\left(\begin{array}{cc}
-i & i \\
1 & 1
\end{array}\right)\left(\begin{array}{cc}
i & 1 \\
-i & 1
\end{array}\right) d\left(\begin{array}{c}
i \\
1
\end{array}\right)-(-i, 1)^{t} b b\left(\begin{array}{c}
i \\
1
\end{array}\right) \\
& =\frac{1}{2}\left(\left(\begin{array}{c}
(i, 1) d\left(\begin{array}{c}
i \\
1
\end{array}\right) \\
(-i, 1) d\left(\begin{array}{c}
i \\
1
\end{array}\right)
\end{array}\right)\right)^{*}\left(\left(\begin{array}{c}
(i, 1) d\left(\begin{array}{c}
i \\
1
\end{array}\right) \\
(-i, 1) d\left(\begin{array}{c}
i \\
1
\end{array}\right)
\end{array}\right)\right)-(-i, 1)^{t} b b\left(\begin{array}{c}
i \\
1
\end{array}\right) .
\end{aligned}
$$


Dividing both sides by $2|\Delta|^{2}=\frac{1}{2}\left|(-i, 1) d\left(\begin{array}{c}i \\ 1\end{array}\right)\right|^{2}$, we get

$$
\left(\left(\begin{array}{c}
{ }^{t} z z \\
1
\end{array}\right)\right)^{*}\left(\left(\begin{array}{c}
t_{z z} \\
1
\end{array}\right)\right)-2 z^{*} z=|\Delta|^{-2} \text {. }
$$

Thus we obtain

$$
|\Delta|^{2}=\frac{1}{1+\left|{ }^{t} z z\right|^{2}-2 z^{*} z}
$$

It follows that

$$
\begin{aligned}
\tau_{\ell}\left(u\left(g k^{-1}\right)\right) & =\left(\frac{1}{2}(-i, 1) d k_{2}^{-1}\left(\begin{array}{c}
i \\
1
\end{array}\right)\right)^{\ell} \\
& =\left(\frac{1}{2}(-i, 1) d\left(\begin{array}{c}
i \\
1
\end{array}\right) e^{i \theta}\right)^{\ell} \\
& =\left(e^{i \theta} \Delta\right)^{\ell} \\
e^{t} & \left.=\mid{ }^{t}(u-z)(u-z)\right) \Delta \mid \\
e^{-2 \rho\left(H\left(g^{-1} k\right)\right)} & =e^{-n t}=\left(\frac{1+\left|{ }^{t} z z\right|^{2}-2 z^{*} z}{|t(u-z)(u-z)|^{2}}\right)^{n / 2} \\
\omega_{\ell, s}\left(\kappa\left(g^{-1} k\right)\right) & =\left(\frac{|t(u-z)(u-z) \Delta|}{e^{-i \theta}(t(u-z)(u-z)) \Delta}\right)^{\ell} \\
\xi_{\ell, s}\left(\exp \left(H\left(g^{-1} k\right)\right)\right) & =\left|{ }^{t}(u-z)(u-z) \Delta\right|^{s}
\end{aligned}
$$

Thus we finally obtain

$$
\begin{aligned}
\mathcal{K}_{\tau_{\ell}, \eta_{\ell, s}}(z, u) & =\tau_{\ell}\left(u\left(g k^{-1}\right)\right) \omega_{\ell, s}\left(\kappa\left(g^{-1} k\right)\right) \xi_{\ell, s}\left(\exp H\left(g^{-1} k\right)\right) e^{-2 \rho\left(H\left(g^{-1} k\right)\right)} \\
& =\left(\frac{e^{2 i \theta}}{t(u-z)(u-z)}\right)^{\ell}\left(\frac{1+\left|{ }^{t} z z\right|^{2}-2 z^{*} z}{\left|{ }^{t}(u-z)(u-z)\right|^{2}}\right)^{(n-\ell-s) / 2}
\end{aligned}
$$

Theorem 6. The generalized Poisson-Cauchy kernel function for Type IV is given by

$$
\mathcal{K}_{\tau_{\ell}, \eta_{\ell, s}}(z, u)=\left(\frac{e^{2 i \theta}}{{ }^{t}(u-z)(u-z)}\right)^{\ell}\left(\frac{1+\left|{ }^{t} z z\right|^{2}-2 z^{*} z}{|t(u-z)(u-z)|^{2}}\right)^{(n-\ell-s) / 2} .
$$

In case $\ell=s=0$ our kernel function coincides with the Poisson kernel function. (See [3], page 99) 
In case $\ell=s=\frac{n}{2}$ our kernel function coincides with the Cauchy kernel function. (See [3], page 97)

\title{
References
}

[1] S. Helgason, Differential Geometry and Symmetric Spaces, Academic Press, Inc, 1962.

[2] S. Helgason, Groups and Geometric Analysis, Academic Press, Inc, 1984.

[3] L.K. Hua, Harmonic analysis of functions of several complex variables in the classical domains, Amer. Math. Soc. (Translations of mathematical monographs), Vol. 6, 1963.

[4] M. Kashiwara, A. Kowata, K. Minemura, K. Okamoto, T. Oshima and M. Tanaka, Eigenfunctions of invariant differential operators on a symmetric space, Ann. of Math., 107 (1978), 1-39.

[5] K. Okamoto, Harmonic analysis on homogeneous vector bundles, Lecture Notes in Math., Springer-Verlag, 266 (1971), 255-271.

[6] K. Okamoto, M. Tsukamoto and K. Yokota, Vector bundle valued Poisson and Cauchy kernel functions on classical domains, to appear in the Proceedings of the Hida Volume: Classical and Quantum White Noise, Kluwer Publishing Co., 2000.

[7] G. Warner, Harmonic analysis of semi-simple Lie groups I, II, Springer-Verlag, BerlinHeidelberg-New York, 1973.

\author{
Kiyosato OKamoto, Michiroh Tsukamoto AND \\ KATSUKI YOKOTA \\ Department of Mathematics \\ FACUlTy OF SCIENCE AND TECHNOLOGY \\ MEIJO UNIVERSITY
}

